ARTICLE

https://doi.org/10.1038/s41467-019-11121-z

\title{
Pioneer and repressive functions of p63 during zebrafish embryonic ectoderm specification
}

\author{
José M. Santos-Pereira (10 ${ }^{1}$, Lourdes Gallardo-Fuentes (1) ${ }^{1}$, Ana Neto ${ }^{1}$, Rafael D. Acemel (D) ${ }^{1}$ \& Juan J. Tena (D) ${ }^{1}$
}

The transcription factor p63 is a master regulator of ectoderm development. Although previous studies show that p63 triggers epidermal differentiation in vitro, the roles of p63 in developing embryos remain poorly understood. Here, we use zebrafish embryos to analyze in vivo how p63 regulates gene expression during development. We generate tp63-knock-out mutants that recapitulate human phenotypes and show down-regulated epidermal gene expression. Following p63-binding dynamics, we find two distinct functions clearly separated in space and time. During early development, p63 binds enhancers associated to neural genes, limiting Sox3 binding and reducing neural gene expression. Indeed, we show that p63 and Sox3 are co-expressed in the neural plate border. On the other hand, p63 acts as a pioneer factor by binding non-accessible chromatin at epidermal enhancers, promoting their opening and epidermal gene expression in later developmental stages. Therefore, our results suggest that p63 regulates cell fate decisions during vertebrate ectoderm specification.

\footnotetext{
${ }^{1}$ Centro Andaluz de Biología del Desarrollo (CABD), Consejo Superior de Investigaciones Científicas/Universidad Pablo de Olavide, 41013 Seville, Spain. Correspondence and requests for materials should be addressed to J.J.T. (email: jjtenagu@upo.es)
} 
T he transcription factor (TF) p63 belongs to the p53 tumorsuppressor protein family and is a master regulator of ectoderm development that is known to play a key role during epidermal specification ${ }^{1,2}$. Its heterozygous mutations in humans have been associated with severe hereditary malformations affecting different ectoderm-derived structures, including skin, palate, digits, hair, and mammary glands ${ }^{3-7}$. These defects correspond to three main phenotypes: split-hand/foot malformation, ectodermal dysplasia, and orofacial cleft ${ }^{8}$. Interestingly, loss-of-function animal models, including mutant mice and knocked-down zebrafish, recapitulate the main human phenotypes, showing absence of skin, limbs and defects in palate development ${ }^{9-12}$.

Recently, the role of p63 over enhancers and genes associated to epidermis differentiation has been highlighted ${ }^{13-18}$, as well as its interaction with chromatin remodeling factors and epigenetic regulators ${ }^{14,19-23}$. The involvement of p63 in enhancing chromatin accessibility in epidermal cells has also been suggested ${ }^{14,16}$, similarly to its related TF $\mathrm{p} 53^{24}$. According to these observations, p63 is a likely candidate to act as a pioneer TF, which are TFs able to bind to non-accessible sites and open them by displacing nucleosomes, alone or assisted by other factors, in order to allow the binding of non-pioneer TFs and other protein ${ }^{25-28}$. Pioneer activity is essential for establishing an active state of chromatin at regulatory regions characterized by nucleosome modifications as H3K4me1 and H3K27ac. However, a pioneer activity of p63 has not been demonstrated so far.

In addition to its role promoting epidermal development, p63 has been involved in the inhibition of other cell fates. In this regard, p63-null mice show an up-regulation of genes required for mesoderm development ${ }^{29,30}$, and zebrafish gastrulating embryos knocked-down for p63 showed an expansion of the neural domains, while p63 over-expression leads to its reduction ${ }^{11,31}$. Interestingly, this work proposed p63 as the neural repressor postulated by the neural default model, according to which ectodermal cells become neural unless inhibited by BMP signaling $^{32}$, and p63 expression is induced by BMP signaling ${ }^{11,18,31}$. However, the mechanism by which p63 might inhibit neuroectoderm expansion is completely unknown.

In this work, we use zebrafish embryos as a model to gain insight into the molecular mechanisms by which $\mathrm{p} 63$ regulates the expression of its target genes. For this, we generate a p63-null zebrafish mutant using CRISPR/Cas9 technology and combine it with the integration of transcriptomic, genomic and epigenomic data. In particular, we use ChIPmentation to analyze the chromatin binding dynamics of $\mathrm{p} 63$ during zebrafish development and find two different functions of p63. During early development, p63 binds to enhancers associated to neural plateexpressing genes, where it limits Sox 3 binding and neural gene expression. Indeed, p63 and Sox 3 are co-expressed at the neural plate border. On the other hand, p63 binds enhancers associated to epidermis-expressing genes when they are in a non-accessible chromatin state, leading to its opening and epidermal gene expression. Therefore, our results suggest that $\mathrm{p} 63$ is an important regulator of ectoderm specification in vertebrates that plays a dual role as a repressor of the neural fate and as a pioneer TF that promotes the epidermal commitment.

\section{Results}

Deregulated epidermal expression in $t p 63^{-/-}$zebrafish mutant. In order to study the effects of the lack of p63 in vertebrate ectoderm development, we generated a tp63 knockout zebrafish model using the CRISPR/Cas9 genome editing system ${ }^{33}$. For this, we targeted the exon 3 of the $\triangle N p 63$ isoforms (exons 5 or 6 of the TAp63 isoforms), generating a 4-bp deletion that lead to a premature stop codon within that exon that affects all tp63 isoforms, leading to the absence of $\mathrm{p} 63$ protein in mutant animals (Fig. 1a). $t p 63^{-9-}$ embryos died just after hatching, between 40 and $50 \mathrm{~h}$ post-fertilization (hpf), and from $36 \mathrm{hpf}$ they showed defects in ectoderm-derived structures, including skin, pectoral fin buds and the fin fold, as reported previously in humans $4,6,7$. From this stage, tp63-1- mutant embryos could be unambiguously identified by observing the lack of pectoral fin buds and the reduced size of the fin fold, while heterozygous $t p 63^{+/-}$embryos showed wild-type phenotype (Fig. 1a, b). In tp63-1- embryos, in situ hybridization of $t b \times 5 a$ shows the formation of the mesodermal anlage that will generate the fin bud, but the apical ectodermal ridge (AER) is not formed and therefore the appendage does not grow (Fig. 1b). Although they were able to grow until more than $40 \mathrm{hpf}$ and pigment cells were less organized but normally visible, the epidermis stopped developing and protecting the animals. Indeed, they died commonly because of a generalized attack by microorganisms or, in a few cases, by neural tissue extrusion. These phenotypes are similar to those described for $\operatorname{trp} 63$ knockout mice, which die after birth due to dehydration and show craniofacial abnormalities, limb truncations as a result of failure of the AER to differentiate, and absence of epidermis and related appendages, including hair follicles, teeth and mammary glands 9,10 . Zebrafish embryos knocked-down for tp63 also showed similar but milder phenotypes ${ }^{11,12}$.

To study the impact of p63 loss at the transcriptomic level, we performed RNA-seq in wild-type and $t p 63^{-/-}$mutant embryos at 36 hpf. Differential gene expression analysis produced 973 upand 1,371 down-regulated transcripts (Fig. 1c). The average foldchange of the down-regulated transcripts was significantly higher than that of the up-regulated ones (Fig. 1d), suggesting a higher global impact of p63 loss over activated genes than over repressed genes at this stage. Consistently with p63 functions, Gene Ontology term enrichment analyses showed biological processes related to epidermis and fin development for the down-regulated genes, and to mesoderm development for the up-regulated ones (Fig. 1e). Moreover, analysis of enrichment of WT expression patterns showed an over-representation of genes expressed in the epidermis for the down-regulated genes and in mesoderm tissues for the up-regulated ones (Fig. 1f). Altogether, these results agree with the positive role of p63 in epidermis and limb development ${ }^{4-7,9,10}$ and with a proposed negative role over mesoderm development ${ }^{29,30}$.

Dynamic p63 binding during ectoderm specification. In order to study in detail how p63 regulates its genetic network during development, we analyzed chromatin binding of p63 by ChIPmentation (ChIP-seq coupled to Tn5-mediated TAGmentation of chromatin $)^{34}$ in different embryonic time-points: $80 \%$ of epiboly (8.3 hpf), $24 \mathrm{hpf}$ and $36 \mathrm{hpf}$, corresponding to gastrulation, segmentation and pharyngula developmental stages, respectively (Fig. 2a, Supplementary Fig. 1). We found a total of 30,597 p63 binding sites (BSs) across the three stages. $K$-means clustering of these BSs produced four clusters ( $\mathrm{a}, \mathrm{b}, \mathrm{c}$ and $\mathrm{d}$ ), two of which showed a clear dynamic behavior: cluster b represented 3,956 early p63 BSs and cluster c was composed of 3,801 late p63 BSs (Fig. 2b). To elucidate whether dynamic p63 BSs were driving expression to different tissues, we assigned them to their putatively regulated genes using GREAT ${ }^{35}$ and performed an analysis of enrichment of WT expression patterns. Interestingly, we found that genes associated to early p63 BSs were mainly expressed in the neural plate, while genes associated to late p63 BSs were preferentially expressed in the epidermis (Fig. 2c). This result suggests that p63 might be regulating gene expression in a tissueand stage-dependent manner through different BSs. 
a

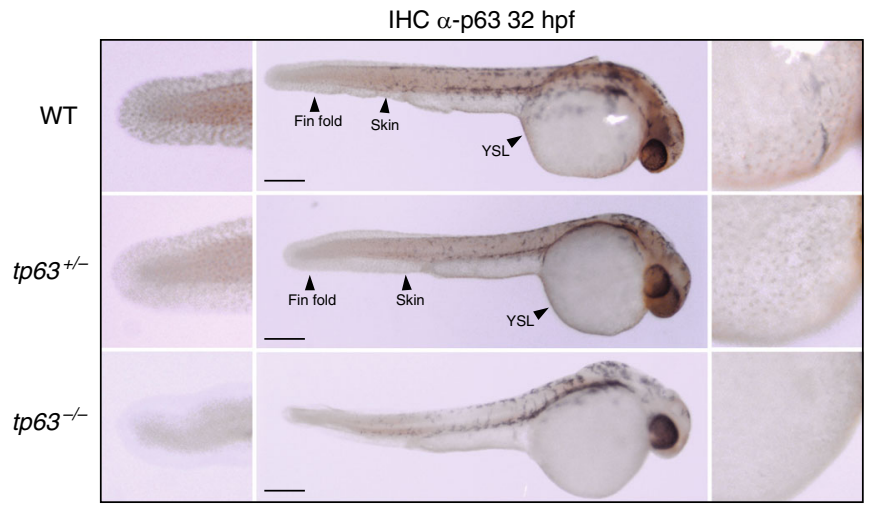

b

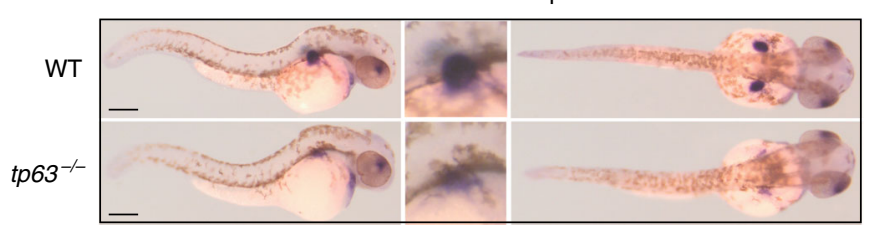

C

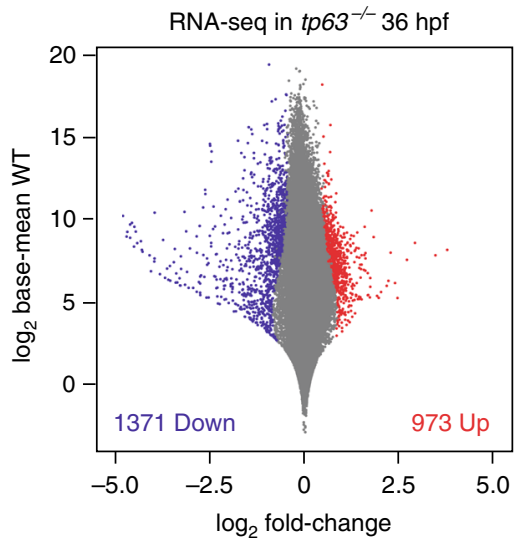

d

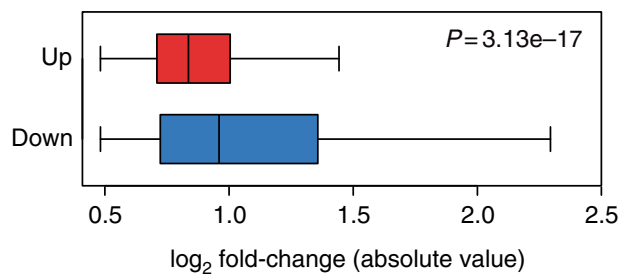

e

GO Biological process term enrichment

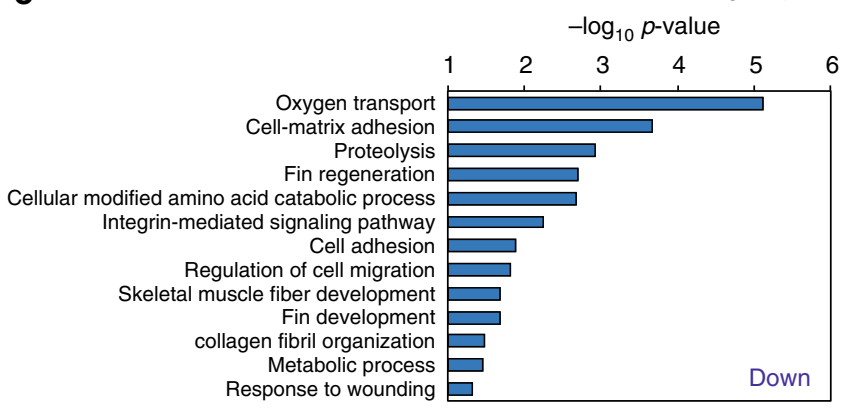

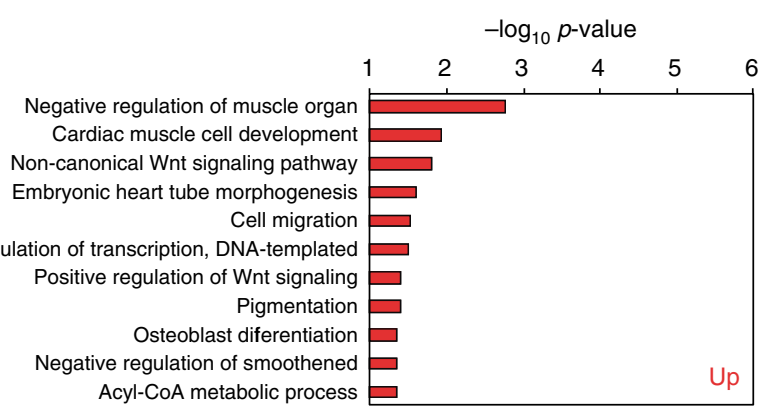

f

Top-10 enrichment of WT expression patterns

$-\log _{10} p$-value

$\begin{array}{llllllllll}1 & 5 & 10 & 15 & 20 & 25 & 30 & 35 & 40 & 45\end{array}$ $-\log _{10} p$-value

$\begin{array}{llllllllll}1 & 5 & 10 & 15 & 20 & 25 & 30 & 35 & 40 & 45\end{array}$

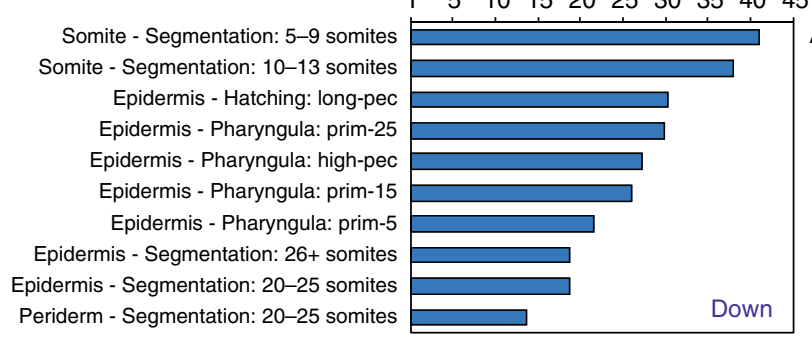

Atrioventric. canal endoc. - Hatching: long-pec
Melanocyte - Pharyngula: prim-5
Melanoblast - Pharyngula: prim-5
Pronephric podocyte - Hatching: long-pec
Melanocyte - Hatching: long-pec
Pronephric glomerulus - Pharyngula: prim-5
Pronephric podocyte - Pharyngula: prim-25
Atrium - Hatching: long-pec
Pronephric podocyte - Pharyngula: prim-5
Proneph glomer. - Larva: protruding mouth

Fig. 1 Zebrafish tp63-/- mutant embryos show de-regulated epidermal program. a Whole-mount embryo immunostaining of p63 in wild-type (WT), heterozygous tp63+/- and homozygous tp63-/- embryos at $32 \mathrm{~h}$ post fertilization (hpf) showing the absence of p63 expression in tp63-/- mutants. Left, zoomed lateral view of the tail; middle, lateral view of whole-embryo; right, zoomed lateral view of the yolk. YSL, yolk syncytial layer. b Whole-mount in situ hybridization of the tbx5a gene in WT and tp63-/- embryos at $36 \mathrm{hpf}$ showing expression in eye, heart and pectoral fin bud, the latter one being reduced in tp63-/- mutant accordingly with the absence of this anatomical structure. Left, lateral view of whole embryo; middle, zoomed lateral view of the pectoral fin bud; right, dorsal view of whole embryo. For $\mathbf{a}$ and $\mathbf{b}$, anterior is to the right. For $\mathbf{a}$ and $\mathbf{b}$, scale bars represent $250 \mu \mathrm{m}$. $\mathbf{c}$ Differential analysis of gene expression between WT and tp63-/- at 36 hpf from RNA-seq ( $n=3$ biological replicates per condition). The $\log _{2}$ base mean WT transcript expression levels versus the $\log _{2}$ fold-change of expression are plotted. Transcripts showing a statistically significant differential expression $(p<0.05)$ are highlighted in red (upregulated) or blue (downregulated). d Box plot representing the absolute $\log _{2}$ fold-change of the up- $(n=973)$ and down-regulated $(n=1,371)$ transcripts from (c). Center line, median; box limits, upper and lower quartiles; whiskers, $1.5 \times$ interquartile range. $p$ value according to the Wilcoxon's rank sum test is shown. Source data are provided as a Source Data file. e Gene Ontology (GO) Biological Process term enrichment of the genes corresponding to the up- and down-regulated transcripts from (c). f Top-10 enrichment of WT expression patterns of the genes corresponding to the up- and downregulated transcripts from (c). For $\mathbf{e}$ and $\mathbf{f}$, the $-\log _{10}$ of $p$-value for each term is shown 
a

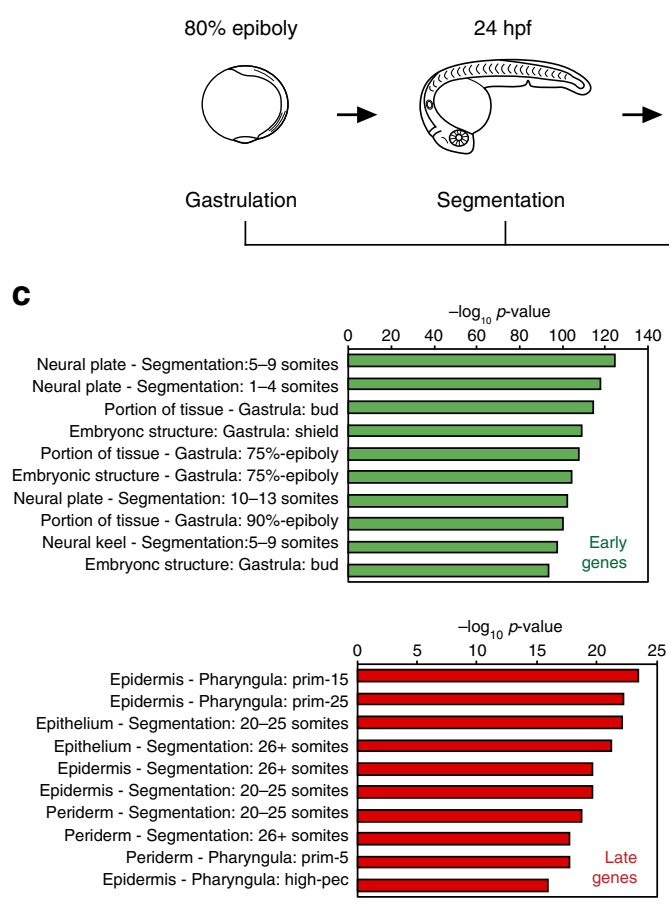

$36 \mathrm{hpf}$

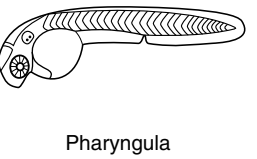

d

Early p63 BSs

PACGCATCTCCGACATCC \#1

ACATTCACACATCTCCE

CCCTCAGACCCAT \#4 TFAP2C 1 1e-106

CCTTTGTT \#6 Sox3 $10-101$

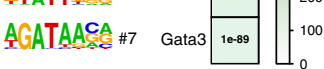

Late $\mathrm{p} 63 \mathrm{BSs}$

AACCATTCC

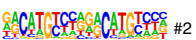

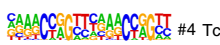

CCACATTCCA \#5 TEAD1 10-107

CCAGGAATCC \#6 TEAD4 b

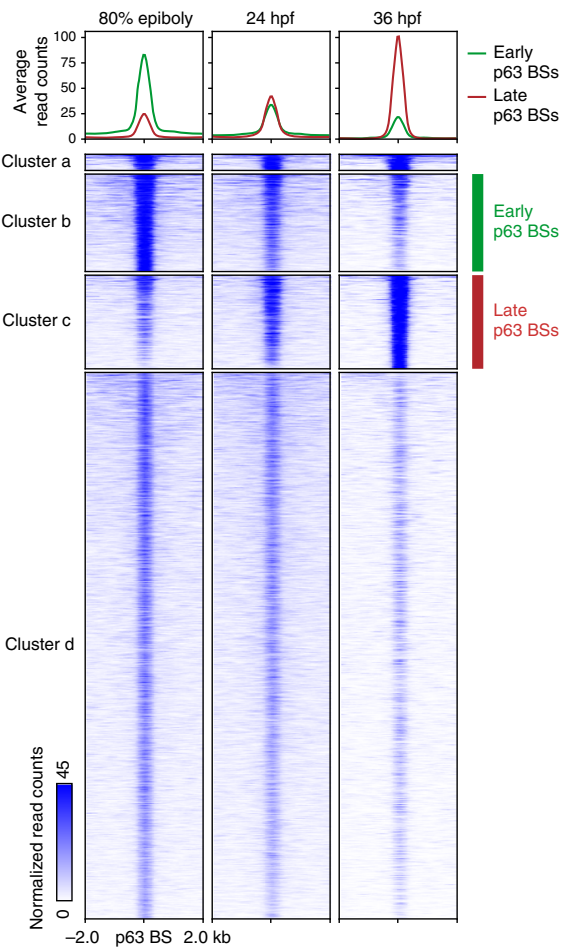

e

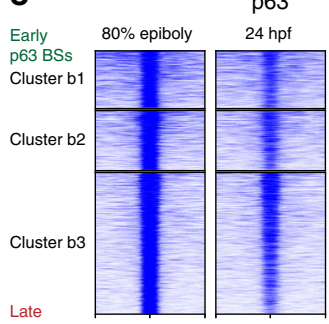

Late
p63 BSs

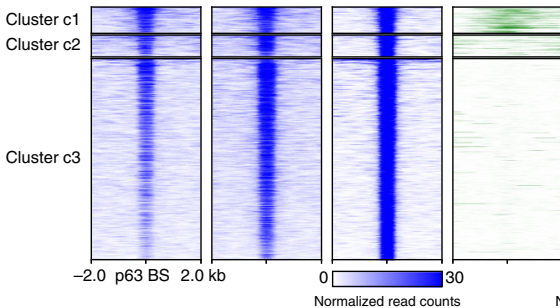

H3K4me3
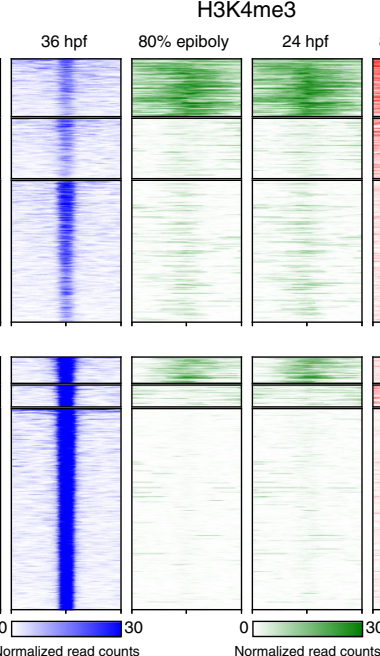

H3K4me1
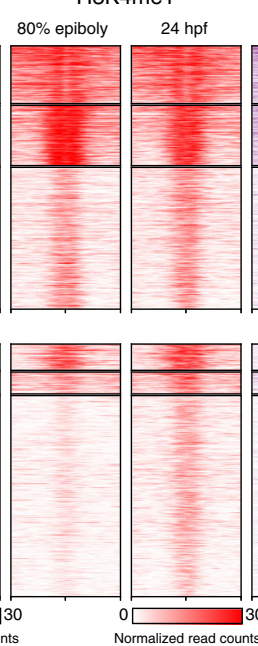

H3K27ac

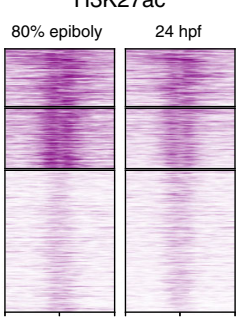

f
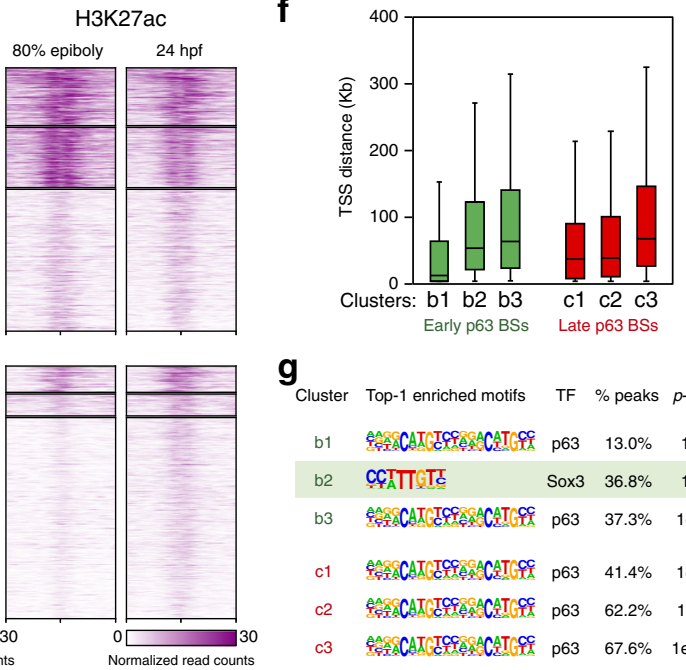

g

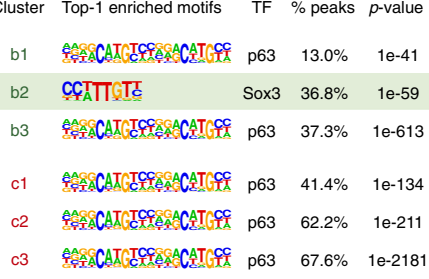

Fig. 2 Dynamics of p63 binding during development in zebrafish embryos. a Picture illustrating zebrafish developmental stages in which most experiments were performed. b Heatmaps of the 30,597 p63 binding sites (BSs) obtained from ChIPmentation in $80 \%$ of epiboly, 24 and 36 hpf stages ( $n=2$ biological replicates per stage). Peaks were clustered using $k$-means clustering, obtaining four groups: cluster a $(n=656)$, cluster b $(n=3,956)$, cluster $\mathrm{c}(n=3,801)$ and cluster $\mathrm{d}(n=22,184)$. Average profiles of cluster b, which was used as the group of early p63 BSs, and cluster c, as late p63 BSs, are shown on top. c Top-10 enrichment of WT expression patterns of the genes corresponding to early (top) and late (bottom) p63 BSs. The - $\log _{10}$ of $p$-value for each term is shown. d Motif enrichment analysis of the early (top) and late (bottom) p63 BSs. Five representative motifs of the top-10 were chosen. Motif logos are represented with their position in the top-10, the transcription factor (TF) name and the enrichment $p$-value in a color scale. e Heatmaps of the early (top) and late (bottom) p 63 BSs clustered according to chromatin marks H3K4me3, H3K4me1 and H3K27ac in $80 \%$ of epiboly and 24 hpf. Peaks were clustered using $k$-means clustering, obtaining three sub-clusters per each group: b1 $(n=875), \mathrm{b} 2(n=912), \mathrm{b} 3(n=2,169), \mathrm{c} 1(n=396), \mathrm{c} 2(n=330)$ and c3 $(n=$ 3,075). $\mathbf{f}$ Box plots showing the distance of the peak sub-clusters from (e) to the transcription start sites (TSS) of the nearby genes. Center line, median; box limits, upper and lower quartiles; whiskers, $1.5 \times$ interquartile range. Source data are provided as a Source Data file. $\mathbf{g}$ Motif enrichment analyses of the peak sub-clusters from (e) representing the most enriched motif in each one, their motif logos, percentage of peaks containing the motif and associated enrichment $p$-value. See also Supplementary Fig. 1

Next, we performed motif enrichment analyses in clusters b and c. The p63 motif was highly enriched in both clusters, validating the ChIP-seq experiment and peak calling analyses (Fig. 2d, Supplementary Fig. 1), together with other motifs of the p53 family (i.e., p53, p73). Motifs of already known partners of p63 were found in the early BSs, like GATA family ${ }^{36}$ or AP2 family ${ }^{37}$ members, but surprisingly also motifs of the Sox family, such as Sox3 and Sox2, which are well known pro-neural TFs. In 
the late BSs, we found members of the TEAD family, which have a role in the maintenance of epidermal progenitors ${ }^{38,39}$ (Fig. 2d, Supplementary Fig. 1). This temporal segregation of p63 partners suggests a sequential switching of the p63 genetic network during development by regulation of different sets of BSs.

We wondered whether epigenomic features could help to functionally catalog dynamic p63 BSs. Therefore, we clustered early and late BSs according to already available epigenomic marks (H3K4me3, H3K4me1 and $\mathrm{H} 3 \mathrm{~K} 27 \mathrm{ac}$ ) in $80 \%$ of epiboly and $24 \mathrm{hpf}$. This clustering produced different groups of peaks corresponding mainly to promoters, with high signal of all the three epigenomic marks (clusters b1 and c1), and enhancers, enriched in $\mathrm{H} 3 \mathrm{~K} 4 \mathrm{me} 1$ and $\mathrm{H} 3 \mathrm{~K} 27 \mathrm{ac}$ signals, but not in $\mathrm{H} 3 \mathrm{~K} 4 \mathrm{me} 3$ (clusters b2, b3, c2 and c3) (Fig. 2e, f, Supplementary Fig. 1). Motif enrichment analyses in these sub-clusters revealed the p63 motif as the most enriched one in all of them, except for cluster b2, in which the most enriched motif was Sox3 (Fig. 2g, Supplementary Fig. 1). This TF is known to be essential for the establishment of neural fate $e^{40,41}$, opposite to the p63 function in the developing embryo. Interestingly, cluster b2 represents early enhancers that are already active in dome stage (blastula, $4.3 \mathrm{hpf}$ ) (Supplementary Fig. 1) and are also enriched in motifs of other Sox family members and pluripotency factors (Supplementary Fig. 1), suggesting that these enhancers are required for the transition from pluripotency to neural fate. This data, the low proportion of BSs showing the p63 motif in this cluster $(11.5 \%$; Supplementary Fig. 1) and the enrichment of neural plate genes associated with early BSs (Fig. 2c), prompted us to explore a possible interaction between p63 and Sox3 that could be important to regulate cell fate during ectoderm specification.

p63 and Sox 3 bind common sites associated to neural genes. p63 has been shown to co-bind common BSs with Sox2 in cancer cells, where they physically interact ${ }^{42,43}$. Moreover, both Sox 2 and Sox 3 bind the same DNA sequence in vitro ${ }^{44}$ and can substitute for each other during embryonic development ${ }^{45}$. To assess whether p63 and Sox3 share some BSs in zebrafish embryos, we performed ChIPmentation assays for Sox 3 in the same developmental stages and compared binding profiles of Sox3 versus p63. Interestingly, a strong overlap was found between both TF BSs, with 18,009 common BSs across the three stages $(22.4 \%$ of total p63 or Sox3 BSs; Fig. 3a), suggesting that p63 and Sox3 might coregulate a subset of common BSs. In agreement with the results mentioned above, these overlapping ChIP-seq peaks showed stronger p63 binding at the early stage (Supplementary Fig. 2a) and were specifically associated to genes enriched in neural plate expression (Fig. 3b, Supplementary Fig. 2b), suggesting that both TFs may cooperate in neural plate gene-associated enhancers.

Motif enrichment analysis of these common BSs showed that the most enriched motifs were those of p63 and Sox3, although the percentage of peaks showing the p63 motif was very low (Fig. 3c). This suggests that p63 could be binding these sites either by direct binding to a different motif or by interaction with another factor, as could be the case of the Sox 3 motif or Sox3 itself. In order to address this question, we analyzed the distribution of distances between the p63 peaks summits (where the TF is presumably located) to the center of the Sox 3 motif at common BSs showing that motif. As a positive control, we compared them with the distances of the p63 summits to the p63 motif in all p63 BSs containing it. Figure 3d shows that p63 summits are indeed centered on the Sox 3 motif in these common BSs, suggesting that p63 binds to the Sox 3 motif rather than near the Sox3 motif.

To further investigate this possibility, we re-analyzed our ChIPmentation data as standard ATAC-seq data, i.e., mapping the precise Tn5 cutting sites. This allowed us to analyze the average footprints generated by p63 and Sox 3 binding to their motifs in different sets of peaks. Indeed, we found that both p63 and Sox3 binding to their respective motifs resulted in a characteristic footprint different to each other (Fig. 3e). Strikingly, plotting the p63 ChIPmentation signal over the Sox3 motif at common BSs revealed a footprint that was weaker but similar to that of Sox3, while Sox3 binding around the p63 motif did not result in any distinguishable footprint (Fig. 3e). These results reinforce the idea that p63 binds to the Sox 3 motif in the BSs shared by both TFs. Although a direct binding of $\mathrm{p} 63$ to the Sox3 motif cannot be discarded, the difference with the footprint generated by p63 over its own motif suggests that p63 binding to Sox 3 motif could occur through interaction with Sox3, which would act as a docking site for p63 at these sites. This hypothesis is also supported by the fact that p63 and Sox2, which is functionally very similar to Sox 3 , bind a subset of common BSs and physically interact in cancer cells $s^{42-45}$.

p63 and Sox 3 are co-expressed at the neural plate border. The genes encoding p63 and Sox3 are expressed in principle in different domains of the developing embryo. During early embryo development, published single-cell RNA-seq (scRNA-seq) data in zebrafish ${ }^{46}$ show that tp63 is expressed at low levels until $60 \%$ of epiboly stage ( $7 \mathrm{hpf}$ ), in which the number of cells showing high tp63 expression increases and remains higher later on, at least until $30 \mathrm{hpf}^{12}$ (Supplementary Fig. 2c). In contrast, sox3 is highly expressed from very early stages (high stage; $3.3 \mathrm{hpf}$ ) and the number of cells showing high expression decreases from $75 \%$ of epiboly stage ( $8 \mathrm{hpf}$ ).

The footprint analysis of p63 binding to Sox 3 motif suggests that p63 and Sox 3 could interact, which would imply that both TFs have to be co-expressed. To check whether there is a population of cells co-expressing them, we first analyzed the scRNA-seq mentioned above ${ }^{46}$ to observe the overlaid expression of tp63 and sox3 during early development. Analysis of the expression patterns of sox 3 and tp63 genes showed that, whereas sox3 expression level was higher in the neuroectoderm and neurogenic placodes branches, tp63 expression was more restricted to the surface ectoderm (Supplementary Fig. 2d), as expected. Interestingly, when we analyzed specifically the bifurcation between the neural and epidermal branches, we found preferential expression of sox 3 in the neural branch and of tp63 in the epidermal one, with a population of intermediate cells co-expressing both genes (Fig. 3f).

To confirm this observation at the protein level, we performed double immunofluorescence assays using specific antibodies against p63 and Sox3 in bud stage zebrafish embryos (10 hpf), when ectoderm tissue is being specified. As expected, we observed expression of Sox 3 in the neuroectoderm and expression of p63 in the surface ectoderm domains, but also a population of cells in the neural plate border expressing both TFs (Fig. 3g). These results demonstrate that Sox 3 and p63 are co-expressed in the neural plate border of zebrafish embryos during early development, where they could be defining the boundary between neuroectoderm and surface ectoderm territories.

p63 regulates neural expression by limiting Sox3 binding. In order to assess whether p63 loss affected Sox3 binding, we took advantage of our tp63 ${ }^{-1-}$ mutant and performed ChIPmentation of Sox3 in 36 hpf-embryos, the earliest stage where the mutant phenotype becomes distinguishable (Supplementary Fig. 3a). Differential Sox3 binding analyses of 147,588 Sox3 BSs between WT and mutant embryos revealed 2,976 up-regulated and 941 downregulated peaks (Fig. 4a, b), suggesting a more prominent role of 


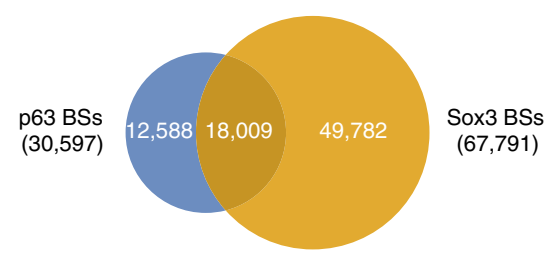

b

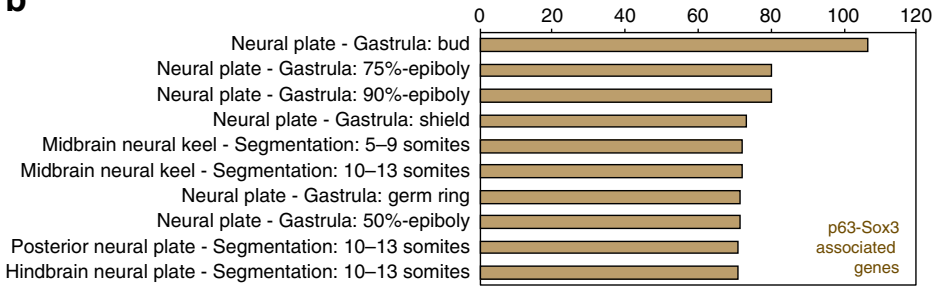

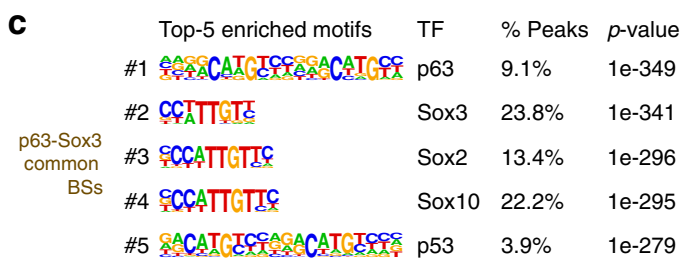

d

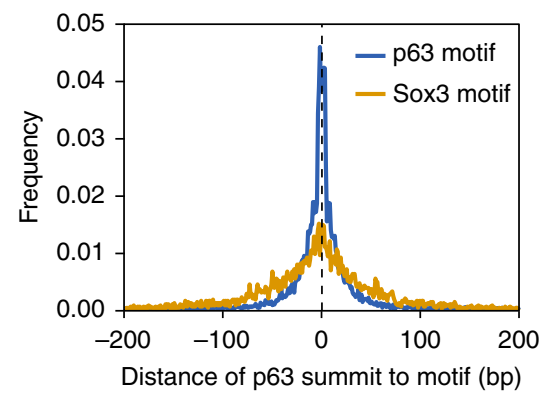

e
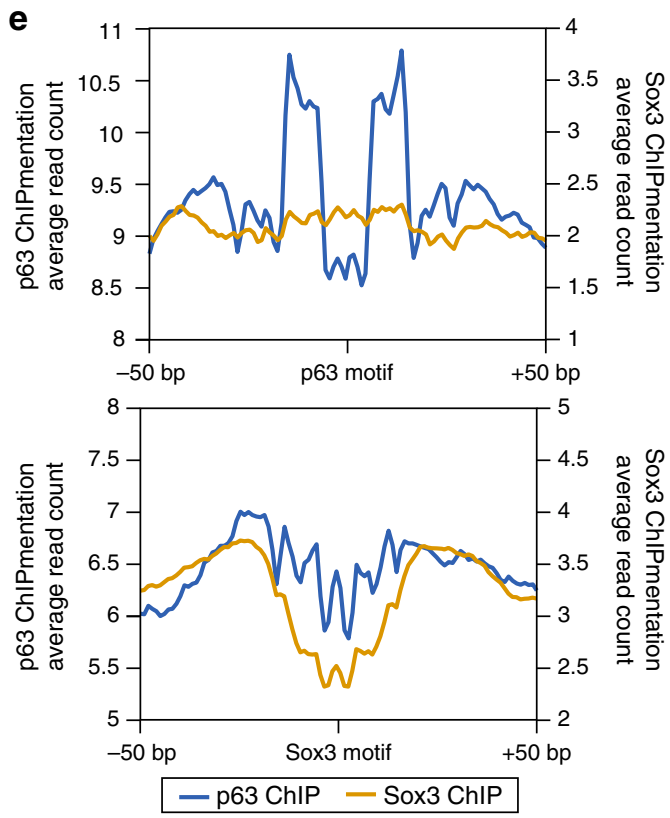

p63 down-regulating Sox3 BSs. $42 \%$ of the up-regulated Sox3 BSs in $t p 63^{-1-}$ mutant overlapped with p63 BSs in WT embryos, but neither of them showed enrichment of the p63 motif (Supplementary Fig. 3b-d). Interestingly, early p63 BSs from our clustering analysis (Fig. 2b) showed enhanced Sox3 signal in the absence of p63, while late p63 BSs did not (Supplementary Fig. 3e), indicating that p63 limits Sox3 binding at early sites enriched in the Sox3 motif. It is worth noting that there is not a detectable change in sox3 expression in $t p 63^{-1-}$ mutant embryos (Fig. 4c), suggesting that p63-dependent inhibition of Sox 3 binding might occur by impeding directly or indirectly that binding.

To check whether up-regulation of Sox3 BSs in the absence of p63 leads to changes in gene expression, we analyzed the subsets

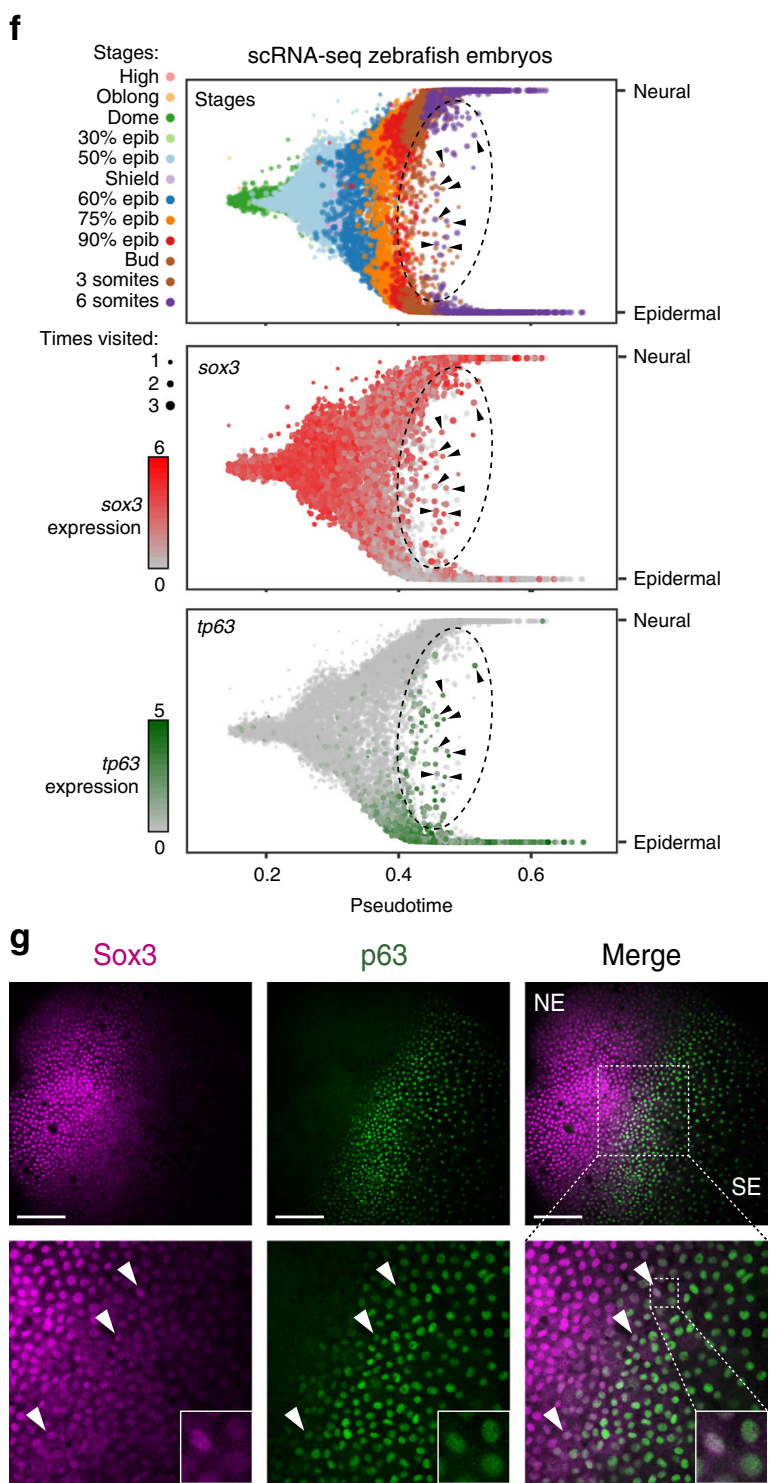

of genes de-regulated in $t p 63^{-/-}$mutant (Fig. 1c) that were also associated to up-, down-regulated or not-changed Sox3 BSs. Strikingly, we found that the expression levels of genes associated to up-regulated Sox3 BSs were also up-regulated in average in tp $63^{-/-}$mutants, while the expression of genes associated to down-regulated or not-changed BSs showed no significant changes compared to all genes (Fig. 4d, e). This suggests that p63 controls the expression of neural genes by regulating Sox3 binding at the nearby enhancers.

P63 binds to epidermal enhancers before chromatin opening. Taking into account that p63 is considered a lineage-determining 
Fig. 3 p63 and Sox3 binding to chromatin and co-expression at the neural plate border. a Venn diagram showing the overlap between p63 and Sox3 peaks from ChIPmentation in $80 \%$ of epiboly, $24 \mathrm{hpf}$ and $36 \mathrm{hpf}$ embryos ( $n=2$ biological replicates per stage). b Top-10 enrichment of WT expression patterns of the genes associated to the common peaks from (a). The - $\log _{10} p$-value for each term is shown. c Motif enrichment analysis of the common peaks from (a). Top-5 motifs are represented with their motif logos, TF name, percentage of peaks containing the motif and enrichment $p$-value. $\mathbf{d}$ Distribution of distances from the p63 ChIP-seq summits to either the p63 or Sox3 motifs in total p63 peaks containing the p63 motif $(n=7,904)$ or in common peaks containing the Sox3 motif $(n=4,286)$, respectively, within a 400-bp window centered in the motifs. e Footprint analysis of p63 and Sox3 binding to their motifs. Average read counts of Tn 5 cutting sites for p63 or Sox3 ChIPmentation signal are plotted centered in either the p63 or Sox3 motifs within a $100-b p$ window. For p63 signal over p63 motif, total p63 BSs containing the p63 motif $(n=7,904)$ were used. For Sox3 signal over Sox3 motif, total Sox3 BSs containing the Sox3 motif $(n=20,817)$ were used. For p63 signal over Sox3 motif and viceversa, common BSs containing the Sox3 ( $n=4,286)$ or the p63 motif $(n=1,645)$, respectively, were used. $\mathbf{f}$ Branchpoint plots of the ectoderm development from scRNA-seq data in zebrafish early development 46 showing pseudotime ( $x$-axis) and random walk visitation preference from the neural to the epidermal domains ( $y$-axis). Top, cells colored by developmental stage. Middle and bottom, gene expression of sox3 and tp63 in red and green, respectively. An ellipse marks the intermediate region between both domains. Arrowheads point to individual cells co-expressing both genes in this intermediate region. $\mathbf{g}$ Double whole-mount embryo immunofluorescence of Sox3 (magenta) and p63 (green) in the neural plate border of bud embryos. Arrowheads point to representative nuclei showing co-expression of both markers. NE neuroectoderm, SE surface ectoderm. Scale bars represent $100 \mu \mathrm{m}$. See also Supplementary Fig. 2

$\mathrm{TF}$ and that a role in regulating chromatin accessibility in epidermal cells has been previously shown ${ }^{14,16,18}$, we wondered whether p63 had the same role in our whole-embryo model. To address this, we compared p63 BSs to ATAC-seq data in $80 \%$ of epiboly and $24 \mathrm{hpf}$ zebrafish embryos. We called p63 and ATACseq peaks in both stages, obtaining a total of 22,583 peaks, and divided them into groups containing a p63 peak, an ATAC-seq peak or both. Then, we plotted the peaks in an alluvial plot to easily visualize transitions between groups across stages (Fig. 5a), excluding the two small groups 2 and 3 . Among the different transitions, we noted that the 1,284 peaks of group 4 were characterized by a very low accessibility at $80 \%$ of epiboly that became higher at $24 \mathrm{hpf}$, combined with high p63 binding in both stages (Fig. 5b, Supplementary Fig. 4a). This suggests that p63 could be acting as a pioneer TF in these regions. Pioneer TFs are able to engage non-accessible sites at condensed chromatin (i.e., closed chromatin) and open them later on in a sequential process, displacing nucleosomes to allow binding of non-pioneer TFs or other proteins ${ }^{25-28}$. Since p63 binds to closed chromatin at $80 \%$ of epiboly stage that becomes open at $24 \mathrm{hpf}$, we will thereafter refer to group 4 as pioneered BSs. Note that group 1 of peaks, representing sites bound by 63 in both stages but without being detected as statistically significant ATAC peaks in neither of them, also shows a weak increase in chromatin opening at $24 \mathrm{hpf}$ (Fig. 5a, b), suggesting that it could represent also sites pioneered by p63 in low abundant cell types. Interestingly, the related TF p53 has been described to have pioneer activity ${ }^{24}$, and this is likely to be the case for p63 as well.

Epigenomic marks of chromatin activation were consistent with chromatin opening of pioneered BSs, being more active at 24 hpf (Fig. 5c, Supplementary Fig. 5a-c), and motif enrichment analysis of these BSs showed that the p63 consensus sequence was the most enriched motif in this group (Fig. 5d). Furthermore, the genes associated to pioneered BSs were preferentially expressed in the epidermis or related tissues, as is the case of lama5 (Fig. 5e, f), suggesting that p63 pioneer function may be specific for epidermal gene-associated enhancers. Note that other groups of peaks from the alluvial analyses did not fit these criteria, except for the group 1 that showed similar features to pioneered BSs (Supplementary Fig. $4 \mathrm{~b}-\mathrm{c}$ and $5 \mathrm{a}-\mathrm{c}$ ). Altogether, our results suggest that p63 may be acting as a pioneer TF over a subset of enhancers associated to epidermal genes.

Pioneer function of p63 promotes epidermal expression. A pioneer function of p63 over epidermal gene-associated enhancers would imply that the lack of p63 impairs its opening. Indeed, a dependency on p63 for the opening of epidermal gene-associated regulatory regions was recently seen in keratinocytes and embryonic skin ${ }^{14,16}$. To test this hypothesis in our whole embryo model, we performed ATAC-seq assays in tp63-/embryos at 8 somites stage, soon after ectoderm-derived tissues are specified, and also at $36 \mathrm{hpf}$, a stage in which neural and epidermal tissues are already formed (Fig. 6a and Supplementary Fig. 5d, e). Comparison of the average chromatin opening at pioneered BSs revealed a decreased ATAC-seq signal in $t p 63^{-1-}$ mutant compared with control embryos (Fig. 6a). This difference was higher in 8 somites than in $36 \mathrm{hpf}$ stage (Supplementary Fig. 5e), probably due to the dilution of the cell population in a more complex embryo. Importantly, the reduction of ATAC-seq signal in $t p 63^{-1-}$ mutant was specific of pioneered BSs, although a decrease was also observed in the group 1 of pioneered-like BSs (Fig. 6a). Therefore, these results indicate that p63 is required for the proper establishment of chromatin accessibility in epidermal gene-associated enhancers.

Then we wondered whether this impairment in chromatin opening had any consequence in the expression of associated genes. We observed particular cases in which pioneered p63 BSs down-regulated in $t p 63^{-/-}$mutant appeared near epidermalexpressing genes, such as collsa1a (Fig. 6b). To test this globally, we compared the fold-change expression of de-regulated genes in tp63-/- mutant associated to pioneered BSs with the rest of deregulated genes in that mutant. Importantly, pioneered BSassociated genes tended to be down-regulated, following the general trend of p63-dependent genes, but this down-regulation was even higher (Fig. 6c). Furthermore, the proportion of downregulated genes was significantly higher for pioneered BSassociated genes than for the rest of genes, switching from a 57 to a $68 \%$ of de-regulated genes (Fig. 6d). Altogether, our results indicate that p63 plays a role as a pioneer TF specifically over epidermal gene-associated enhancers, promoting the expression of epidermal genes.

According to our data, we propose a model in which p63 plays a dual role in vivo during ectoderm specification, with two distinct functions that are temporally, spatially and mechanistically separated (Fig. 6e). During gastrulation, p63 prevents Sox3 binding to early enhancers in the neural plate border, leading to the repression of the neural transcriptomic program. This interaction may limit the expansion of the neural plate into the epidermal domains and promote epidermal specification. On the other hand, p63 binds to non-accessible chromatin at enhancers associated to epidermal genes, promoting their opening by a pioneer function and activating the epidermal transcriptomic program during epidermis differentiation at segmentation and pharyngula stages. The dual role proposed is consistent with previous reports that show activation of the epidermal lineage genetic program by 63 in different systems through chromatin opening $1,14,16,18$, and neural expansion during early development for down-regulation of $\mathrm{p} 63$ in different animal 
a

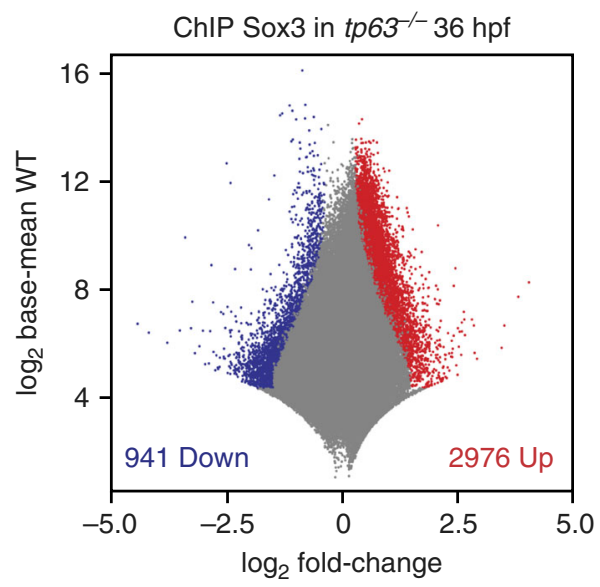

b

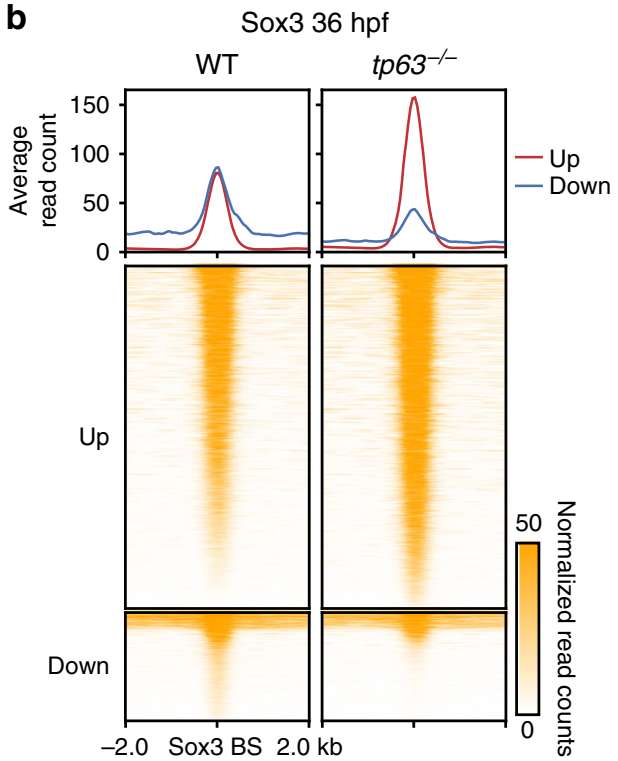

C

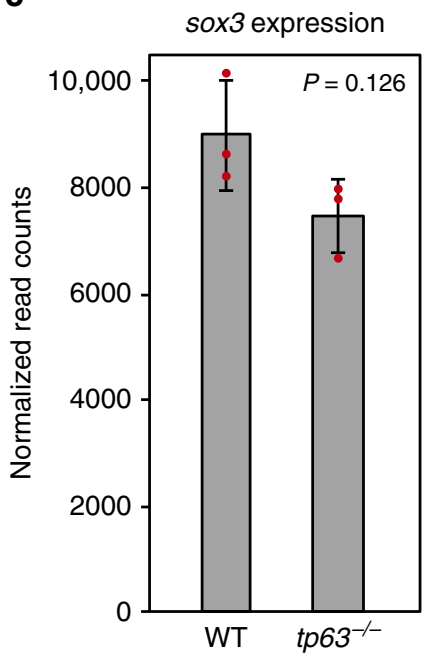

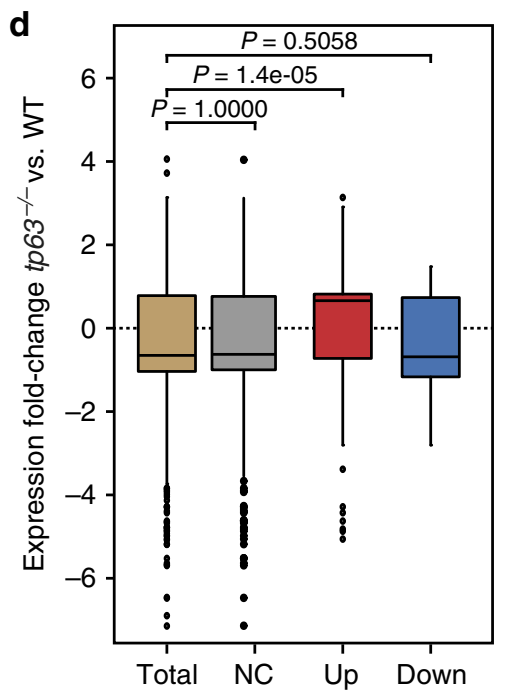

$\mathbf{e}$

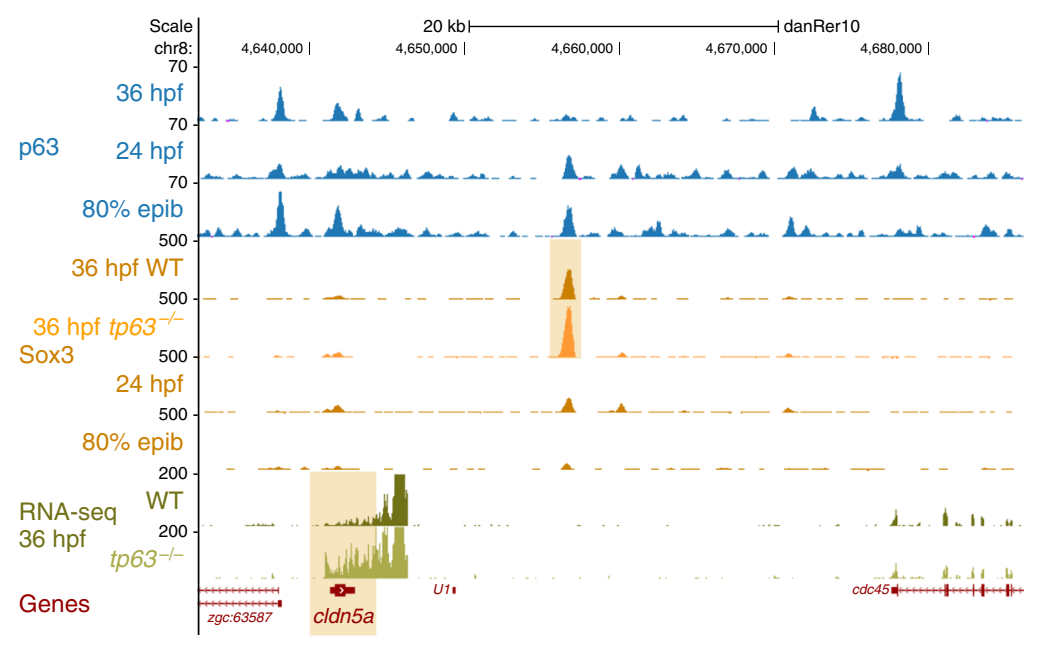

Fig. 4 p63 regulates Sox3 binding to neural enhancers and the neural program. a Differential analysis of Sox3 binding between WT and tp63-/- at 36 hpf calculated in a set of 147,588 Sox3 BSs (FDR < 0.05) from ChIPmentation ( $n=2$ biological replicates per condition). The log 2 base mean Sox3 binding levels in the WT versus the $\log _{2}$ fold-change of expression are plotted. Peaks showing a statistically significant differential expression $(p<0.01)$ are highlighted in blue (downregulated) or red (upregulated). b Heatmaps and average profiles of the Sox3 BSs at 36 hpf from (a) in WT and tp63-/embryos. Peaks were separated according to the differential analysis in up-regulated (Up; $n=2,976$ ) or down-regulated (Down; $n=941)$ in tp63-/mutant compared to WT. c Bar plot with the mean expression of sox3 gene in WT vs. tp63-/- embryos at 36 hpf as measured by RNA-seq ( $n=3$, for each condition). Error bars represent s.d. Individual values are shown as red dots. Non-corrected $p$-value from the differential expression analysis is shown. d Box plots showing the expression fold-change in tp63-/- embryos of the transcripts associated to Up- $(n=222)$, Down-regulated $(n=75)$ and not changed (NC; $n=1,255)$ Sox3 BSs from (a) compared to the total de-regulated transcripts $(n=2,344)$ in that mutant. Center line, median; box limits, upper and lower quartiles; whiskers, 1.5× interquartile range; points, outliers. Statistical significance was assessed using the Wilcoxon's rank sum test. For (c) and (d), source data are provided as a Source Data file. e Genome tracks of p63 ChIP-seq, Sox3 ChIP-seq, and RNA-seq in the indicated developmental stages showing signal intensities in a region of chromosome 8. An up-regulated Sox3 BS in tp63-/- mutant and the associated up-regulated gene (cldn5a) are highlighted in light orange. The Genes track represents ENSEMBL annotated genes. See also Supplementary Fig. 3

models ${ }^{11,31}$. Further work will be necessary to understand the molecular mechanisms by which p63 inhibits DNA binding of neural TFs, as Sox3, to neural gene-associated enhancers, as well as the specific role of p63 partners, as AP2, GATA or TEAD family members, during epidermal commitment.

\section{Methods}

Animal experimentation. Wild-type $\mathrm{AB} /$ Tübingen zebrafish strains were maintained and bred under standard conditions. All experiments involving animals conform national and European Community standards for the use of animals in experimentation and were approved by the Ethical Committees from the University Pablo de Olavide, CSIC and the Andalusian government.

CRISPR-Cas9 genomic edition. CRISPR target site to mutate the tp63 gene was identified using the CRISPRscan online tool ${ }^{47,48}$. A small guide RNA (sgRNA) targeting the exon 3 of the $\Delta N p 63$ variants (exons 5 or 6 of TAp63 variants) was used with the following target sequence: $5^{\prime}$-GCC CGT ATG ACT GCA CCC TGG GG-3'. The template DNA for sgRNA transcription was generated by PCR using primers sgRNA_tp63_exon3 and sgRNA_universal (Supplementary Table 1) with Phusion DNA polymerase (Thermo Fisher Scientific). sgRNA was in vitro transcribed using the HiScribe T7 Quick High Yield RNA synthesis kit (NEB) using 


\section{a}

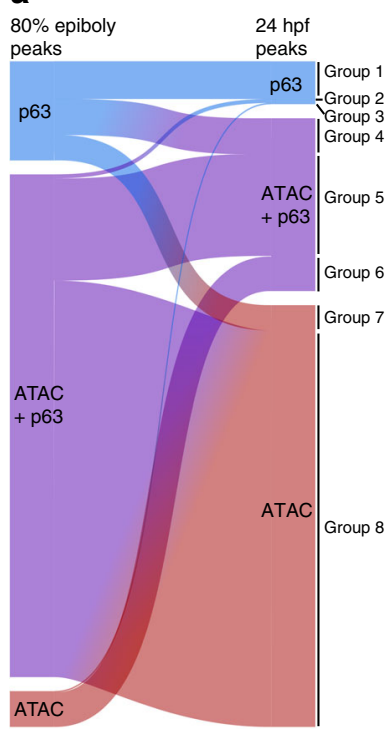

e

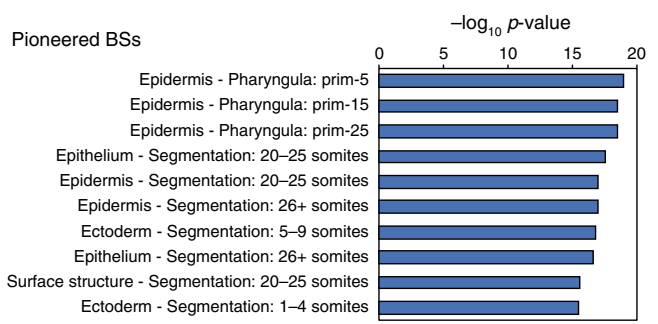

b

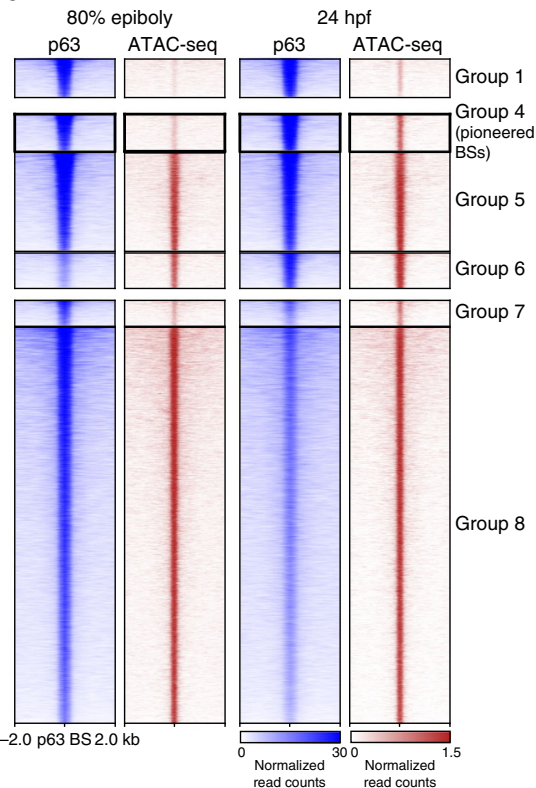

C

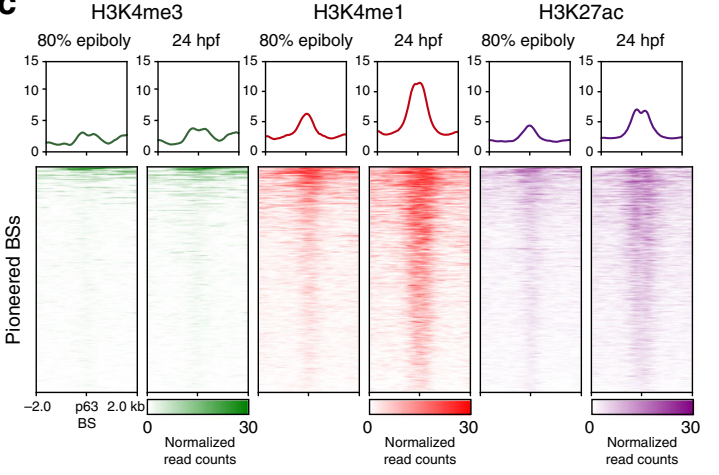

d

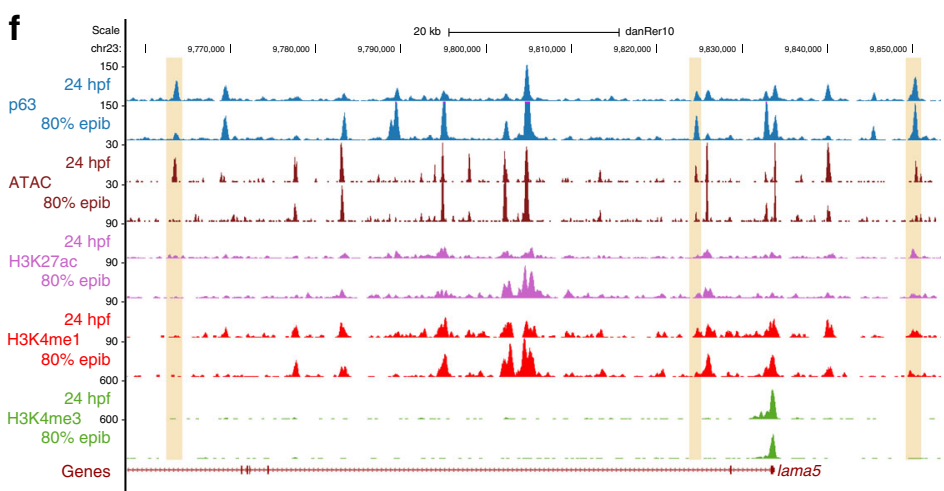

Fig. 5 p63 binding to epidermal enhancers precedes chromatin opening. a Alluvial plot showing the transition between $80 \%$ of epiboly and 24 hpf stages of the 22,583 peaks showing p63 binding (blue), chromatin accessibility (ATAC; red) or both (purple). Peaks showing no p63 binding in any of the two analyzed developmental stages were not considered. The transitions lead to eight groups of p63 BSs: group 1 ( $n=332$ peaks), group 2 ( $n=145$ peaks), group 3 ( $n=43$ peaks), group 4 ( $n=1,284$ peaks), group 5 ( $n=3,619$ peaks), group $6(n=1,227$ peaks), group 7 ( $n=896$ peaks) and group 8 ( $n=$ 14,037). b Heatmaps of p63 and ATAC-seq signal in $80 \%$ of epiboly and 24 hpf stages for the groups resulting from the alluvial plot transitions in (a). Groups 2 and 3 were omitted due to their small number of peaks. c Heatmaps and average profiles of H3K4me3, H3K4me1 and H3K27ac in $80 \%$ of epiboly and $24 \mathrm{hpf}$ for the group 4 of peaks from (a) (pioneered p63 BSs). d Motif enrichment analysis of the pioneered p63 BSs. The top-5 motifs are represented with their motif logos, position in the top-5, the TF name and the enrichment $p$-value in a color scale. e Top-10 enrichment of WT expression patterns for the genes corresponding to the pioneered p63 BSs. The - $\log _{10}$ of $p$-value for each term is shown. $\mathbf{f}$ Genome tracks of p63 ChIP-seq, ATACseq, H3K27ac, H3K4me1, and H3K4me3 in the indicated developmental stages showing signal intensities in a region of chromosome 23. Pioneered p63 BSs associated to the epidermis-expressed gene lama5 are highlighted in light orange. The Genes track represents ENSEMBL annotated genes. See also Supplementary Fig. 4

$75 \mathrm{ng}$ of template, treated with DNase I (NEB) and purified using the RNA Clean and Concentrator kit (Zymo Research).

One-cell stage zebrafish embryos were co-injected with $2-3 \mathrm{~nL}$ of a solution containing $300 \mathrm{ng} / \mu \mathrm{L}$ Cas 9 protein and $20 \mathrm{ng} / \mu \mathrm{L}$ sgRNA, pre-incubated during $10 \mathrm{~min}$ on ice. The CRISPR-Cas9 genomic edition generated a deletion of $4 \mathrm{bp}\left(5^{\prime}\right.$ GTGC-3'; mutation tp63 $\triangle 4$ ) resulting in a premature STOP codon in the same exon. For screening of the edited genome, genomic DNA was obtained by incubating the samples (whole embryos or adult caudal fin fragments) in TE buffer supplemented with $5 \%$ Chelex-100 (BioRad) and $10 \mu \mathrm{g} / \mathrm{mL}$ Proteinase K (Roche) for $1 \mathrm{~h}$ (embryos) or $4 \mathrm{~h}$ (fins) at $55^{\circ} \mathrm{C}$ and $10 \mathrm{~min}$ at $95^{\circ} \mathrm{C}$, and then stored at $4{ }^{\circ} \mathrm{C}$ One microliter of the supernatant was used as a template for standard $25-\mu \mathrm{L}$ PCR reactions using primers tp63-ex3-Fw and tp63-ex3-Rv (Supplementary Table 1). The resulting 338-bp (334-bp for tp63 44 alleles) amplicons were digested with PasI restriction enzyme, since the 4-bp deletion resulted in loss of the PasI site and consequently the enzyme digestion generates 260 and 78-bp bands in WT alleles

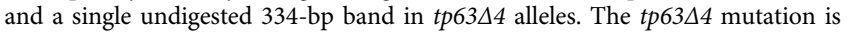

stably maintained in heterozygosis with no apparent phenotypes, as homozygous mutants are embryonic lethal ( $<3$ days).

Whole-mount embryo immunostaining. For immunostaining, embryos were fixed overnight at $4{ }^{\circ} \mathrm{C}$ with $4 \%$ paraformaldehyde, washed in PBT (PBS supplemented with $0.2 \%$ Triton-X100) and blocked in this solution with $2 \%$ goat serum and $2 \mathrm{mg} / \mathrm{mL}$ BSA for $1 \mathrm{~h}$ at RT. Then, they were incubated overnight at $4{ }^{\circ} \mathrm{C}$ with primary antibody. Primary polyclonal antibodies were anti-Tp63 (used 1:100 dilution, GTX124660 GeneTex) for p63 immunohistochemistry, or anti-p63 (used 1:50 diltuion, 4A4 Abcam) and anti-Sox3 (used 1:100 dilution, GTX132494 GeneTex) for p63 and Sox3 immunofluorescence. After extensive washings with PBT, embryos were incubated overnight at $4^{\circ} \mathrm{C}$ with anti-rabbit secondary antibodies conjugated with Hrp (used 1:500 dilution, NA934 Sigma Aldrich) for p63 immunohistochemistry, or goat anti-mouse conjugated with Alexa Fluor 488 (used 1:800 dilution, A28175 Invitrogen) or donkey anti-rabbit conjugated with Alexa Fluor 555 (used 1:500 dilution, A31572 Invitrogen) for p63 and Sox3 


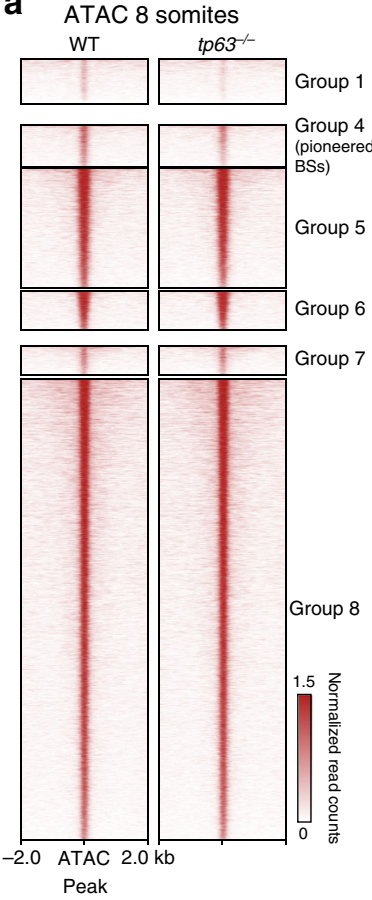

ATAC 8 somites

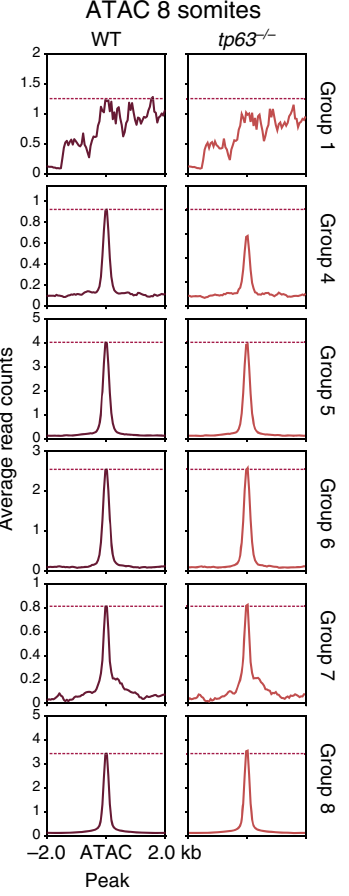

b

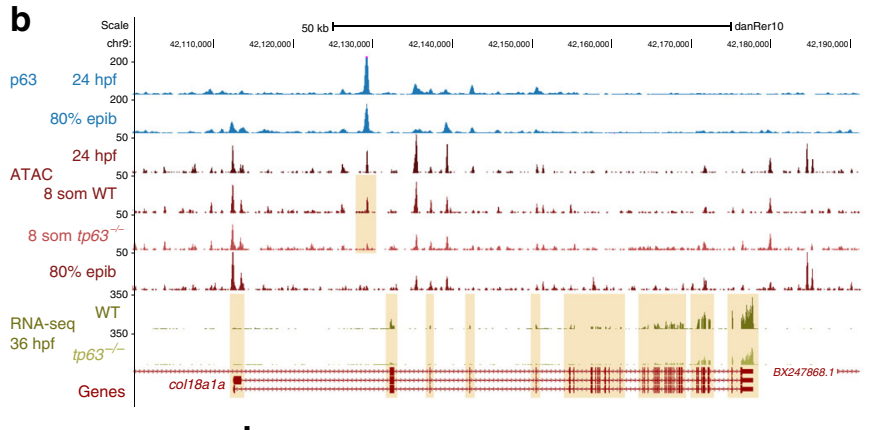

C

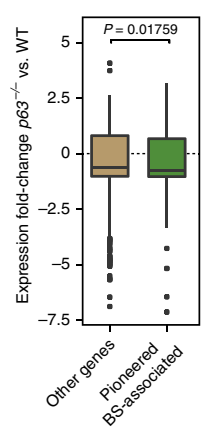

d

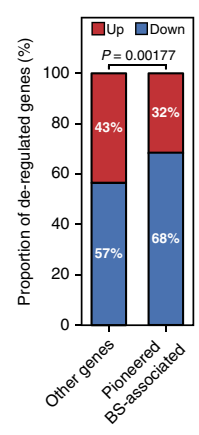

e

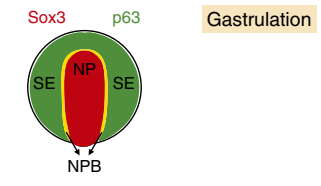

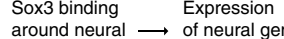
around neural $\longrightarrow$ of neural genes
genes

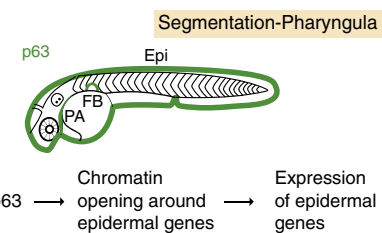

Fig. 6 p63 acts as a pioneer TF to promote epidermal gene expression. a Heatmaps (left) and average profiles (right) of ATAC-seq signal from WT and tp63 ${ }^{-/-}$embryos in 8 somites stage $(n=2$ biological replicates per condition) for the groups resulting from the alluvial plot transitions from Fig. 5 a. A dotted red line represents the WT profile peak in each stage. Groups 2 and 3 were omitted from the analyses due to their small number of peaks. b Genome tracks of p63 ChIP-seq, ATAC-seq and RNA-seq in the indicated developmental stages showing signal intensities in a region of chromosome 9. A down-regulated ATAC-seq peak in tp63-/- mutant and the associated down-regulated gene (col18a1a) are highlighted in light orange. The Genes track represents ENSEMBL annotated genes. c Box plots showing the expression fold-change in tp63-/- embryos of the transcripts associated to group 4 ATACseq peaks $(n=227)$ compared to the remaining de-regulated transcripts in that mutant $(n=2,117)$. Center line, median; box limits, upper and lower quartiles; whiskers, $1.5 \times$ interquartile range; points, outliers. Statistical significance was assessed using the Wilcoxon's rank sum test. Source data are provided as a Source Data file. d Proportion of up- and down-regulated transcripts in tp63- $/$ - embryos associated to ATAC-seq peaks from group 4 compared to the remaining de-regulated transcripts in that mutant. Statistical significance was assessed using the Fisher's exact test. e Model proposed to illustrate the dual role of p63 during ectoderm specification. During gastrulation (top), p63 prevents Sox3 binding to enhancers associated to neural genes probably in the neural plate border, where they are co-expressed, resulting in the inhibition of neural expression. During segmentation and pharyngula (bottom), non-accessible chromatin at enhancers associated to epidermal genes and bound by p63 during gastrulation becomes open, triggering an epidermal transcriptomic program that results in the differentiation of epidermis and related structures. NP neural plate, SE surface ectoderm, NPB neural plate border, Epi epidermis, FB fin bud, PA pharyngeal arches. See also Supplementary Fig. 5

immunofluorescence, respectively. For immunohistochemistry, embryos were whole-mounted in agarose and imaged under a MZ-12 dissecting scope (Leica). For immunofluorescence, embryos were flat-mounted and imaged under an SP confocal microscope (Leica).

Whole-mount embryo in situ hybridization. Antisense RNA probe against $t b x 5 a$ gene was prepared from cDNA using digoxigenin (Boehringer Mannheim) as label. Zebrafish embryos were prepared, hybridized and stained using standard proto$\operatorname{cols}^{49}$. Embryos at $36 \mathrm{hpf}$ stage were fixed in $4 \%$ paraformaldehyde overnight, dehydrated in methanol and stored at $-20^{\circ} \mathrm{C}$. All solutions and reagents used were RNAse-free. The embryos were hydrated using decreasing amounts of methanol and finally in PBS- $0.1 \%$ Tween. Then, they were treated with $10 \mu \mathrm{g} / \mathrm{mL}$ proteinase $\mathrm{K}$ for $10^{\prime}$ at room temperature and gently washed with PBS- $0.1 \%$ Tween. In the pre-hybridization step, embryos were kept at $70^{\circ} \mathrm{C}$ in the hybridization buffer for at least $1 \mathrm{~h}$. Then, the probe was diluted to $2 \mathrm{ng} / \mu \mathrm{L}$ in hybridization buffer and incubated overnight at $70^{\circ} \mathrm{C}$ with movement. Pre-heated buffers with decreasing amounts of hybridization buffer $(75,50,25$, and $0 \%)$ in $2 \times$ SSC solution were used to wash embryos for $10^{\prime}$, plus a $30^{\prime}$ wash at $70^{\circ} \mathrm{C}$ with $0.05 \times$ SSC. Then, they were incubated with Blocking Buffer (PBS- $0.1 \%$ Tween, $2 \%$ normal goat serum, $2 \mathrm{mg} /$ $\mathrm{mL}$ bovine serum albumin [BSA]) for $1 \mathrm{~h}$, and with antibody anti-digoxigenin (1:5000 in Blocking Buffer) for at least $2 \mathrm{~h}$ at room temperature. After this, embryos were washed six times with PBS- $0.1 \%$ Tween at room temperature and then overnight at $4{ }^{\circ} \mathrm{C}$. Next day, embryos were washed once more with PBS- $0.1 \%$ Tween and three times with fresh AP buffer $(100 \mathrm{mM}$ Tris- $\mathrm{HCl} \mathrm{pH} \mathrm{9.5,50} \mathrm{mM}$ $\mathrm{MgCl}_{2}, 100 \mathrm{mM} \mathrm{NaCl}, 0.1 \%$ Tween), followed by signal revelation with NBT/BCIP solution ( $225 \mu \mathrm{g} / \mathrm{mL} \mathrm{NBT}, 175 \mu \mathrm{g} / \mathrm{mL}$ BCIP) in multi-well plates in the dark. After tracking the developing signal, the revelation was stopped by washing with
PBS- $0.1 \%$ Tween and fixing with $4 \%$ paraformaldehyde. Imaging of the in situ hybridization signal was performed in MZ-12 dissecting scope (Leica).

RNA-seq. For total RNA extraction, fifteen WT and fifteen $t p 63^{-/-}$embryos in $36 \mathrm{hpf}$ stage per experiment were collected, manually de-chorionated and suspended in TRIsure (Bioline) with chloroform. Precipitated RNA was then treated with TURBO DNA free kit (Invitrogen). In parallel, DNA was extracted to genotype pooled embryos. Three biological replicates were used for each analyzed line Illumina libraries were constructed and sequenced in a BGISEQ-500 single-end lane producing around $50 \mathrm{M}$ of 50-bp reads. Reads were aligned to the GRCz10 (danRer10) zebrafish genome assembly using STAR $2.5 .3 \mathrm{a}^{50}$ and counted using the htseq-count tool from the HTSeq 0.8.0 toolkit ${ }^{51}$. Differential gene expression analysis was performed using the DESeq2 1.18 .1 package in $\mathrm{R} 3.4 .3^{52}$, setting a corrected $p$ value $<0.05$ as the cutoff for statistical significance of the differential expression. Enrichment of GO Biological Process term was calculated using David ${ }^{53}$, with a Bonferroni-corrected $p$ value $<0.05$ as statistical cutoff. Enrichment of wild-type zebrafish expression patterns was calculated by a custom script (Supplementary Software 1), using a two-tailed hypergeometric test with a Bonferroni-corrected $p$ value $<0.05$ as statistical cutoff.

Single-cell RNA-seq data analyses. Single-cell RNA-seq data analyses from zebrafish embryos were performed over published data using the URD package ${ }^{46}$ in R 3.4.3. Developmental trajectories were calculated between the epidermal and neural branches of the ectoderm specification (69 and 70 nodes, respectively), considering as epidermal the tips 1 and 10, and as neural the tips 2, 8, 16, 19, 50, 56, and 59 (see ref. ${ }^{46}$ ). 
ATAC-seq. ATAC-seq assays were performed using standard protocols $s^{54,55}$, with minor modifications. Briefly, pools of $5 \mathrm{WT}$ or $t p 63^{-/-}$mutant embryos at $36 \mathrm{hpf}$, or single 8 somites-stage zebrafish embryos coming from $t p 63^{+/-}$parents, were manually de-chorionated. Yolk was dissolved with Ginzburg Ring Finger $(55 \mathrm{mM}$ $\mathrm{NaCl}, 1.8 \mathrm{mM} \mathrm{KCl}, 1.15 \mathrm{mM} \mathrm{NaHCO}$ ) by pippeting and shaking 5 ' at 1,100 rpm. Deyolked embryos were collected by centrifugation for $5^{\prime}$ at $500 \times g 4^{\circ} \mathrm{C}$. Supernatant was removed and embryos washed with PBS. Then, embryos were lysed in $50 \mu \mathrm{L}$ of Lysis Buffer (10 mM Tris- $\mathrm{HCl} \mathrm{pH} \mathrm{7.4,} 10 \mathrm{mM} \mathrm{NaCl}, 3 \mathrm{mM} \mathrm{MgCl}, 0.1 \%$ NP-40, $1 \times$ Roche Complete protease inhibitors cocktail) by pippeting up and down. For 8-somites embryos, the whole cell lysate was used for the next step; for 36-hpf embryos, half of the sample was used to count the cell number in a Neubauer chamber with Hoechst staining, and about 75,000 cells were used. For TAGmentation, cells were centrifuged for $10^{\prime}$ at $500 \mathrm{~g} 4^{\circ} \mathrm{C}$ and resuspended in 50 $\mu \mathrm{L}$ of the Transposition Reaction, containing $1.25 \mu \mathrm{L}$ of Tn5 enzyme and TAGmentation Buffer $(10 \mathrm{mM}$ Tris- $\mathrm{HCl} \mathrm{pH} 8.0,5 \mathrm{mM} \mathrm{MgCl} 2,10 \% \mathrm{w} / \mathrm{v}$ dimethylformamide), and incubated for $30^{\prime}$ at $37^{\circ} \mathrm{C}$. Immediately after TAGmentation, DNA was purified using the Minelute PCR Purification Kit (Qiagen), and eluted in $20 \mu \mathrm{L}$. Before library amplification, purified DNA was used to genotype 8-somites embryos (see above) and two WT and two tp63 $3^{-/}$mutants were selected for deep sequencing. Libraries were generated by PCR amplification using NEBNext HighFidelity 2X PCR Master Mix (NEB). The resulting library was multiplexed and sequenced in a HiSeq 4000 pair-end lane producing $100 \mathrm{M}$ of 49 -bp pair end reads, or in a NextSeq 500 pair-end lane producing $100 \mathrm{M}$ of 51-bp pair end reads. ATAC-seq assays of $80 \%$ epiboly and $24 \mathrm{hpf}$ embryos were previously reported $^{56-58}$. Reads were aligned using the GRCz10 (danRer10) zebrafish genome assembly and those pairs separated by more than $2 \mathrm{~Kb}$ were removed. The Tn5 cutting site was determined as the position -4 (minus strand) or +5 (plus strand) from each read start, and this position was extended $5 \mathrm{bp}$ in both directions. The IDR framework (idr 0.1 version) was used to obtain high confidence peaks based on replicate information, as previously described ${ }^{58}$. Reads were extended $100 \mathrm{bp}$ only for visualization in the UCSC Genome Browser ${ }^{59}$.

For data comparison, all ATAC-seq experiments used (two replicates for each stage, including 8 somites and $36 \mathrm{hpf}$ experiments, as well as previously published $80 \%$ epiboly and $24 \mathrm{hpf}$ experiments) were normalized using reads falling into peaks to counteract differences in background levels between experiments and replicates. For this, peaks were first called for each experiment using the IDR framework (see above) and all peaks merged in a single file. Then, the number of reads falling into those peaks was determined for each experiment and the dataset with less reads into peaks was taken as a reference. Finally, the number of total random reads to be taken from each experiment was estimated considering the minimal number of reads into peaks to be taken and the percentage of reads into peaks of each one.

ChIPmentation. ChIP-seq of p63 and Sox 3 were performed by ChIPmentation, which incorporates Tn5-mediated TAGmentation of immunoprecipitated DNA ${ }^{34}$. Briefly, 3,000 zebrafish embryos in $80 \%$ epiboly stage or 1,000 in 24 or 36 hpf stages (120 embryos for Sox 3 ChIP in $t p 63^{-/-}$mutants) were dechorionated with $300 \mu \mathrm{g} /$ $\mathrm{mL}$ pronase, fixed for $10 \mathrm{~min}$ in $1 \%$ paraformaldehyde (in $200 \mathrm{mM}$ phosphate buffer) at RT, quenched for $5 \mathrm{~min}$ with $0.125 \mathrm{M}$ glycine, washed in PBS and frozen at $-80^{\circ} \mathrm{C}$. Fixed embryos were homogenized in $5 \mathrm{~mL}$ cell lysis buffer $(10 \mathrm{mM}$ Tris$\mathrm{HCl}$ pH 7.5, $10 \mathrm{mM} \mathrm{NaCl}, 0.3 \% \mathrm{NP}-40,1 \times$ Roche Complete protease inhibitors cocktail) ( $2 \mathrm{~mL}$ for ChIP in tp63-/- mutants) with a Dounce Homogenizer on ice and centrifuged $5 \mathrm{~min} 2,300 \times \mathrm{g}$ at $4^{\circ} \mathrm{C}$. Pelleted nuclei were resuspended in $1,320 \mu \mathrm{L}$ of nuclear lysis buffer (50 mM Tris-HCl pH 7.5, $10 \mathrm{mM}$ EDTA, $1 \%$ SDS, $1 \times$ Roche Complete protease inhibitors cocktail) $(333 \mu \mathrm{L}$ for ChIP in tp63-1mutants), kept $5 \mathrm{~min}$ on ice and diluted with $2,680 \mu \mathrm{L}$ of ChIP dilution buffer (16.7 mM Tris-HCl pH 7.5, 1.2 mM EDTA, $167 \mathrm{mM} \mathrm{NaCl}, 0.01 \%$ SDS, $1.1 \%$ Triton-X100) (667 $\mu \mathrm{L}$ for ChIP in tp63-1- mutants). Then, chromatin was sonicated in a Covaris M220 sonicator (duty cycle 10\%, PIP $75 \mathrm{~W}, 100$ cycles/burst, $10 \mathrm{~min}$ ) or in a Bioruptor 200 sonicator (High intensity, 30-s ON-30-s OFF, $15 \mathrm{~min}$ ) and centrifuged $5 \mathrm{~min} 18,000 \times g$ at $4^{\circ} \mathrm{C}$. The recovered supernatant, which contained soluble chromatin, was used for ChIP or frozen at $-80^{\circ} \mathrm{C}$ after checking the size of the sonicated chromatin. Six $200-\mu \mathrm{L}$ aliquots of sonicated chromatin (four $250-\mu \mathrm{L}$ aliquots for ChIP in $t p 63^{-/-}$mutants) were used for each independent ChIP experiment, and each aliquot incubated with $2 \mu \mathrm{g}$ of anti-p63 (GTX124660 GeneTex) or anti-Sox3 (GTX132494 GeneTex) antibodies and rotated overnight at $4{ }^{\circ} \mathrm{C}$.

Next day, $20 \mu \mathrm{L}$ of protein G Dynabeads (Invitrogen) per aliquot were washed twice with ChIP dilution buffer and resuspended in $50 \mu \mathrm{L} /$ aliquot of the same solution. Immunoprecipitated chromatin was then incubated with washed beads for $1 \mathrm{~h}$ rotating at $4{ }^{\circ} \mathrm{C}$ and washed twice sequentially with wash buffer $1(20 \mathrm{mM}$ Tris-HCl pH 7.5, 2 mM EDTA, $150 \mathrm{mM} \mathrm{NaCl}, 1 \%$ SDS, $1 \%$ Triton-X100), wash buffer $2(20 \mathrm{mM}$ Tris-HCl pH 7.5, $2 \mathrm{mM}$ EDTA, $500 \mathrm{mM} \mathrm{NaCl}, 0.1 \%$ SDS, $1 \%$ Triton-X100), wash buffer 3 (10 mM Tris- $\mathrm{HCl} \mathrm{pH} \mathrm{7.5,} 1 \mathrm{mM}$ EDTA, $250 \mathrm{mM} \mathrm{LiCl}$, $1 \% \mathrm{NP}-40,1 \% \mathrm{Na}$-deoxycholate) and $10 \mathrm{mM}$ Tris- $\mathrm{HCl} \mathrm{pH} \mathrm{8.0,} \mathrm{using} \mathrm{a} \mathrm{cold} \mathrm{magnet}$ (Invitrogen). Then, beads were resuspended in $25 \mu \mathrm{L}$ of TAGmentation reaction mix (10 mM Tris- $\mathrm{HCl} \mathrm{pH} \mathrm{8.0,} 5 \mathrm{mM} \mathrm{MgCl} 2,10 \% \mathrm{w} / \mathrm{v}$ dimethylformamide), added $1 \mu \mathrm{L}$ of $\mathrm{Tn} 5$ enzyme and incubated $1 \mathrm{~min}$ at $37^{\circ} \mathrm{C}$. TAGmentation reaction was put in the cold magnet and the supernatant discarded. Beads were washed twice again with wash buffer 1 and $1 \times \mathrm{TE}$, and eluted twice for $15 \mathrm{~min}$ in $100 \mu \mathrm{L}$ of elution buffer (50 mM NaHCO3 pH 8.8, 1\% SDS). The $200 \mu \mathrm{L}$ of eluted chromatin per aliquot were then decrosslinked by adding $10 \mu \mathrm{L}$ of $4 \mathrm{M} \mathrm{NaCl}$ and $1 \mu \mathrm{L}$ of $10 \mathrm{mg}$ / $\mathrm{mL}$ proteinase $\mathrm{K}$ and incubating at $65^{\circ} \mathrm{C}$ for $6 \mathrm{~h}$. DNA was purified using Minelute PCR Purification Kit (Qiagen), pooling all aliquots in a single column, and eluted in $20 \mu \mathrm{L}$.

Library preparation was performed as previously described for ATAC-seq ${ }^{54,55}$ (see above). Libraries were multiplexed and sequenced in a HiSeq 4000 pair-end lane producing around $20 \mathrm{M}$ of 49 -bp pair end reads. Reads were aligned to the GRCz10 (danRer10) zebrafish genome assembly using Bowtie ${ }^{60}$. Peaks were called using MACS2 algorithm ${ }^{61}$ with an FDR $<0.001$. ChIP-seq of H3K4me3, H3K4me1 and H3K27ac were previously reported ${ }^{62}$. For data comparison, ChIP-seq experiments using the same antibody, two replicates of each stage, except for Sox 3 in $80 \%$ epiboly and $24 \mathrm{hpf}$, were normalized by taking an equal number of random total reads, which were extended 300 bp for data visualization.

ChIPmentation and ATAC-seq data analyses. Conversion of SAM alignment files to BAM was performed using Samtools ${ }^{63}$. Conversion of BAM to BED files, and peak analyses, such as overlaps or merges, were carried out using the Bedtools suite $^{64}$. Conversion of BED to BigWig files was performed using the genomecov tool from Bedtools and the wigToBigWig utility from UCSC. Heatmaps and average profiles of ChIP-seq and ATAC-seq data were generated using computeMatrix, plotHeatmap and plotProfile tools from the Deeptools 2.0 toolkit ${ }^{65}$. Correlation analyses were performed using only the reads falling into peaks $(p<0.001)$ and empirically excluding peaks with abnormally high number of reads. $k$-means clustering was performed when required using Deeptools 2.0 or seqMiner ${ }^{66}$ (rank clustering method). Differential analysis of Sox 3 peaks in WT versus tp $63^{-1-}$ mutant was performed from the complete set of 147,588 peaks from the four experiments called with MACS2 (FDR $<0.05$ ), using DESeq2 1.18 .1 package ${ }^{52}$ in $\mathrm{R}$ 3.4.3. A corrected $p$ value $<0.01$ was set as the cutoff for statistical significance of the differential expression analysis. TF motif enrichment was calculated using the script FindMotifsGenome.pl from Homer software ${ }^{67}$, with standard parameters.

For the footprinting analysis, peaks containing the p63 or Sox 3 motifs were obtained using the annotatePeaks.pl tool from Homer software. The Tn5 cutting sites were determined as the position -4 (minus strand) or +5 (plus strand) from each read start, and this position was extended $5 \mathrm{bp}$ in both directions, as described above for ATAC-seq. Finally, average read counts around p63 or Sox 3 motifs in a 100 -bp window centered in the motifs were plotted.

For gene assignment to ChIP and ATAC peaks, coordinates were converted to $\mathrm{Zv} 9$ (danRer7) genome using the Liftover tool of the UCSC Genome Browser ${ }^{59}$ and assigned to genes using the GREAT tool ${ }^{35}$, with the basal plus extension association rule with standard parameters $(5 \mathrm{~Kb}$ upstream, $1 \mathrm{~Kb}$ downstream, $1 \mathrm{Mb}$ maximum extension). Enrichment of wild-type zebrafish expression patterns was calculated by GREAT with standard parameters (significant by both region-based binomial and gene-based hypergeometric test, FDR $<0.05$ ).

Statistical analyses. For comparison between expression fold-changes among multiple gene sets, the Kruskal-Wallis rank sum test was first used to test for differences among groups, followed by pairwise comparisons using a two-tailed Wilcoxon's rank sum test. For comparison between expression levels of two gene sets, a two-tailed Wilcoxon's rank sum test was used. In both cases, box plots represent: center line, median; box limits, upper and lower quartiles; whiskers, $1.5 \times$ interquartile range; points, outliers. Statistical significance of contingency tables was assessed using the Fisher's exact test.

Reporting summary. Further information on research design is available in the Nature Research Reporting Summary linked to this article.

\section{Data availability}

ChIPmentation, ATAC-seq and RNA-seq data generated in this study are available through the Gene Expression Omnibus under accession code GSE123059. Public datasets used in this study are available from GEO: GSE32483 (ChIP-seq of histone modifications), GSE61065 (ATAC-seq $24 \mathrm{hpf}$ ) and GSE106428 (ATAC-seq 80\% of epiboly). The source data underlying Figs. 1d, 2f, $4 \mathrm{c}, 4 \mathrm{~d}$ and $6 \mathrm{c}$ are provided as a Source Data File.

\section{Code availability}

Custom code used in this study is available as a Supplementary Software 1. All the data analyses were performed using publicly available software.

Received: 20 December 2018 Accepted: 20 June 2019 Published online: 11 July 2019

\section{References}

1. Soares, E. \& Zhou, H. Master regulatory role of p63 in epidermal development and disease. Cell. Mol. Life Sci. 75, 1179-1190 (2018). 
2. Yang, A. et al. P63, a P53 homolog at 3Q27-29, encodes multiple products with transactivating, death-inducing, and dominant-negative activities. Mol. Cell 2, 305-316 (1998).

3. van Bokhoven, H. et al. p63 Gene mutations in EEC syndrome, limbmammary syndrome, and isolated split hand-split foot malformation suggest a genotype-phenotype correlation. Am. J. Hum. Genet. 69, 481-492 (2001).

4. Celli, J. et al. Heterozygous germline mutations in the p53 homolog p63 are the cause of EEC syndrome. Cell 99, 143-153 (1999).

5. Rinne, T., Hamel, B., Van Bokhoven, H. \& Brunner, H. G. Pattern of p63 mutations and their phenotypes - Update. Am. J.Med. Genet. Part A 140, 1396-1406 (2006).

6. Amiel, J. et al. TP63 gene mutation in ADULT syndrome. Eur. J. Hum. Genet. 9, 642-645 (2001).

7. Ianakiev, P. et al. Split-hand/split-foot malformation is caused by mutations in the p63 gene on 3q27. Am. J. Hum. Genet. 67, 59-66 (2000).

8. Rinne, T., Brunner, H. G. \& Van Bokhoven, H. P63-associated disorders. Cell Cycle 6, 262-268 (2007).

9. Mills, A. A. et al. P63 is a P53 homologue required for limb and epidermal morphogenesis. Nature 398, 708-713 (1999)

10. Yang, A. et al. p63 is essential for regenerative proliferation in limb, craniofacial and epithelial development. Nature 398, 714-718 (1999).

11. Bakkers, J., Hild, M., Kramer, C., Furutani-Seiki, M. \& Hammerschmidt, M. Zebrafish $\Delta \mathrm{Np} 63$ is a direct target of Bmp signaling and encodes a transcriptional repressor blocking neural specification in the ventral ectoderm. Dev. Cell 2, 617-627 (2002).

12. Lee, H. \& Kimelman, D. A dominant-negative form of $\mathrm{p} 63$ is required for epidermal proliferation in zebrafish. Dev. Cell 2, 607-616 (2002).

13. Kouwenhoven, E. N. et al. Transcription factor p63 bookmarks and regulates dynamic enhancers during epidermal differentiation. EMBO Rep. 16, 863-878 (2015).

14. Bao, X. et al. A novel ATAC-seq approach reveals lineage-specific reinforcement of the open chromatin landscape via cooperation between BAF and p63. Genome Biol. 16, 284 (2015).

15. Sethi, I., Sinha, S. \& Buck, M. J. Role of chromatin and transcriptional coregulators in mediating p63-genome interactions in keratinocytes. BMC Genom. 15, 1042 (2014).

16. Fan, X. et al. Single cell and open chromatin analysis reveals molecular origin of epidermal cells of the skin. Dev. Cell 47, 21-37.e5 (2018).

17. Li, L. et al. TFAP2C- and p63-dependent networks sequentially rearrange chromatin landscapes to drive human epidermal lineage commitment. Cell Stem Cell 24, 271-284.e8 (2019).

18. Pattison, J. M. et al. Retinoic acid and BMP4 cooperate with p63 to alter chromatin dynamics during surface epithelial commitment. Nat. Genet. 50, 1658-1665 (2018).

19. Mardaryev, A. N. et al. p63 and Brg1 control developmentally regulated higher-order chromatin remodelling at the epidermal differentiation complex locus in epidermal progenitor cells. Development 141, 3437-3437 (2014).

20. Mardaryev, A. N. et al. Cbx4 maintains the epithelial lineage identity and cell proliferation in the developing stratified epithelium. J. Cell Biol. 212, 77-89 (2016).

21. Fessing, M. Y. et al. P63 regulates Satb1 to control tissue-specific chromatin remodeling during development of the epidermis. J. Cell Biol. 194, 825-839 (2011).

22. Lin-Shiao, E. et al. KMT2D regulates p63 target enhancers to coordinate epithelial homeostasis. Genes Dev. 32, 181-193 (2018).

23. Rinaldi, L. et al. Dnmt3a and Dnmt3b associate with enhancers to regulate human epidermal stem cell homeostasis. Cell Stem Cell 19, 491-501 (2016).

24. Sammons, M. A., Zhu, J., Drake, A. M. \& Berger, S. L. TP53 engagement with the genome occurs in distinct local chromatin environments via pioneer factor activity. Genome Res. 25, 179-188 (2015).

25. Cirillo, L. A. et al. Opening of compacted chromatin by early developmental transcription factors HNF3 (FoxA) and GATA-4. Mol. Cell 9, 279-289 (2002).

26. Zaret, K. S. \& Mango, S. E. Pioneer transcription factors, chromatin dynamics, and cell fate control. Curr. Opin. Genet. Dev. 37, 76-81 (2016).

27. Magnani, L., Eeckhoute, J. \& Lupien, M. Pioneer factors: directing transcriptional regulators within the chromatin environment. Trends Genet. 27, 465-474 (2011).

28. Mayran, A. \& Drouin, J. Pioneer transcription factors shape the epigenetic landscape. J. Biol. Chem. 293, 13795-13804 (2018).

29. Shalom-Feuerstein, R. et al. $\Delta$ np63 Is an ectodermal gatekeeper of epidermal morphogenesis. Cell Death Differ. 18, 887-896 (2011).

30. De Rosa, L. et al. P63 suppresses non-epidermal lineage markers in a bone morphogenetic protein-dependent manner via repression of Smad7. J. Biol. Chem. 284, 30574-30582 (2009).

31. Tríbulo, C. et al. $\triangle \mathrm{Np} 63$ is regulated by BMP4 signaling and is required for early epidermal development in Xenopus. Dev. Dyn. 241, 257-269 (2012).

32. Hemmati-Brivanlou, A. \& Melton, D. Vertebrate embryonic cells will become nerve cells unless told otherwise. Cell 88, 13-17 (1997).
33. Jinek, M. et al. A programmable dual-RNA-guided DNA endonuclease in adaptive bacterial immunity. Science 337, 816-821 (2012).

34. Schmidl, C., Rendeiro, A. F., Sheffield, N. C. \& Bock, C. ChIPmentation: fast, robust, low-input ChIP-seq for histones and transcription factors. Nat. Methods 12, 963-965 (2015).

35. Hiller, M. et al. Computational methods to detect conserved non-genic elements in phylogenetically isolated genomes: Application to zebrafish. Nucleic Acids Res. 41, e151 (2013).

36. Candi, E. et al. p63 is upstream of IKK in epidermal development. J. Cell Sci. 119, 4617-4622 (2006).

37. McDade, S. S. et al. Genome-wide analysis of p63 binding sites identifies AP-2 factors as co-regulators of epidermal differentiation. Nucleic Acids Res. 40, 7190-7206 (2012).

38. Zhang, H., Pasolli, H. A. \& Fuchs, E. Yes-associated protein (YAP) transcriptional coactivator functions in balancing growth and differentiation in skin. Proc. Natl Acad. Sci. USA 108, 2270-2275 (2011).

39. Schlegelmilch, K. et al. Yap1 acts downstream of $\alpha$-catenin to control epidermal proliferation. Cell 144, 782-795 (2011).

40. Dee, C. T. et al. Sox3 regulates both neural fate and differentiation in the zebrafish ectoderm. Dev. Biol. 320, 289-301 (2008).

41. Rogers, C. D., Harafuji, N., Archer, T., Cunningham, D. D. \& Casey, E. S Xenopus Sox 3 activates sox 2 and geminin and indirectly represses Xvent2 expression to induce neural progenitor formation at the expense of non-neural ectodermal derivatives. Mech. Dev. 126, 42-55 (2009).

42. Watanabe, H. et al. SOX2 and p63 colocalize at genetic loci in squamous cell carcinomas. J. Clin. Invest 124, 1636-1645 (2014).

43. Jiang, Y. et al. Co-activation of super-enhancer-driven CCAT1 by TP63 and SOX2 promotes squamous cancer progression. Nat. Commun. 9, 3619 (2018).

44. Kamachi, Y., Cheah, K. S. E. \& Kondoh, H. Mechanism of regulatory target selection by the SOX high-mobility-group domain proteins as revealed by comparison of SOX1/2/3 and SOX9. Mol. Cell. Biol. 19, 107-120 (2015).

45. Adikusuma, F., Pederick, D., McAninch, D., Hughes, J. \& Thomas, P. Functional equivalence of the SOX2 and SOX3 transcription factors in the developing mouse brain and testes. Genetics 206, 1495-1503 (2017).

46. Farrell, J. A. et al. Single-cell reconstruction of developmental trajectories during zebrafish embryogenesis. Science 360, eaar3131 (2018).

47. Moreno-Mateos, M. A. et al. CRISPRscan: designing highly efficient sgRNAs for CRISPR-Cas9 targeting in vivo. Nat. Methods 12, 982-988 (2015).

48. Vejnar, C. E., Moreno-Mateos, M. A., Cifuentes, D., Bazzini, A. A. \& Giraldez, A. J. Optimization strategies for the CRISPR-Cas9 genome-editing system. Cold Spring Harb. Protoc. 2016, 829-832 (2016).

49. Tena, J. J. et al. Odd-skipped genes encode repressors that control kidney development. Dev. Biol. 301, 518-531 (2007).

50. Dobin, A. et al. STAR: ultrafast universal RNA-seq aligner. Bioinformatics 29, 15-21 (2013).

51. Anders, S., Pyl, P. T. \& Huber, W. HTSeq-a Python framework to work with high-throughput sequencing data. Bioinformatics 31, 166-169 (2015).

52. Love, M. I., Huber, W. \& Anders, S. Moderated estimation of fold change and dispersion for RNA-seq data with DESeq2. Genome Biol. 15, 550 (2014).

53. Huang, D. W., Sherman, B. T. \& Lempicki, R. A. Systematic and integrative analysis of large gene lists using DAVID bioinformatics resources. Nat. Protoc. 4, 44-57 (2009).

54. Buenrostro, J. D., Giresi, P. G., Zaba, L. C., Chang, H. Y. \& Greenleaf, W. J. Transposition of native chromatin for fast and sensitive epigenomic profiling of open chromatin, DNA-binding proteins and nucleosome position. Nat. Methods 10, 1213-1218 (2013).

55. Fernández-Miñán, A., Bessa, J., Tena, J. J. \& Gómez-Skarmeta, J. L. Assay for transposase-accessible chromatin and circularized chromosome conformation capture, two methods to explore the regulatory landscapes of genes in zebrafish. Methods Cell Biol. 135, 413-430 (2016).

56. Gehrke, A. R. et al. Deep conservation of wrist and digit enhancers in fish. Proc. Natl Acad. Sci. USA 112, 803-808 (2015).

57. Bogdanović, O. et al. Active DNA demethylation at enhancers during the vertebrate phylotypic period. Nat. Genet. 48, 417-426 (2016).

58. Marlétaz, F. et al. Amphioxus functional genomics and the origins of vertebrate gene regulation. Nature 564, 64-70 (2018).

59. Casper, J. et al. The UCSC Genome Browser database: 2018 update. Nucleic Acids Res. 46, D762-D769 (2018).

60. Langmead, B. \& Salzberg, S. L. Fast gapped-read alignment with Bowtie 2. Nat. Methods 9, 357-359 (2012).

61. Zhang, Y. et al. Model-based analysis of ChIP-Seq (MACS). Genome Biol. 9, R137 (2008).

62. Bogdanović, O. et al. Dynamics of enhancer chromatin signatures mark the transition from pluripotency to cell specification during embryogenesis. Genome Res. 22, 2043-2053 (2012). 
63. Li, H. et al. The sequence alignment/map format and SAMtools. Bioinformatics 25, 2078-2079 (2009).

64. Quinlan, A. R. \& Hall, I. M. BEDTools: a flexible suite of utilities for comparing genomic features. Bioinformatics 26, 841-842 (2010).

65. Ramírez, F. et al. deepTools2: a next generation web server for deepsequencing data analysis. Nucleic Acids Res. 44, W160-W165 (2016).

66. Ye, T. et al. seqMINER: An integrated ChIP-seq data interpretation platform. Nucleic Acids Res. 39, e35-e35 (2011).

67. Heinz, S. et al. Simple combinations of lineage-determining transcription factors prime cis-regulatory elements required for macrophage and B cell identities. Mol. Cell 38, 576-589 (2010).

\section{Acknowledgements}

We thank J.L. Gómez-Skarmeta, J.R. Martínez-Morales and I. Maeso for critical reading of the manuscript; the CABD Fish Facility for technical assistance; S. Muñoz-Galván and A. Carnero for providing reagents; and C3UPO for the HPC support. This work was supported by a grant from the Spanish Ministry of Economy and Competitiveness to J.J.T. (BFU2014-58449-JIN) and an ERC-Advanced grant from the European Research Council (ERC) under the European Union's Horizon 2020 research and innovation program (grant agreement No. 740041). J.M.S.-P. was funded by a postdoctoral contract associated to an Excellence Project from Junta de Andalucía (P12-BIO-396) and by a Juan de la Cierva-Incorporación fellow from the Spanish Ministry of Economy and Competitiveness (IJCI-2016-29884).

\section{Author contributions}

J.J.T. and J.M.S.-P. conceived and designed the project; J.M.S.-P., L.G.-F., and A.N. performed the experiments; J.M.S.-P., R.D.A., and J.J.T. analyzed the data; J.J.T. and J.M.S.-P. wrote the manuscript.

\section{Additional information}

Supplementary Information accompanies this paper at https://doi.org/10.1038/s41467019-11121-z.

Competing interests: The authors declare no competing interests.

Reprints and permission information is available online at http://npg.nature.com/ reprintsandpermissions/

Peer review information: Nature Communications thanks Xiaomin Bao and other anonymous reviewer(s) for their contribution to the peer review of this work. Peer reviewer reports are available.

Publisher's note: Springer Nature remains neutral with regard to jurisdictional claims in published maps and institutional affiliations.

(c) (i) Open Access This article is licensed under a Creative Commons Attribution 4.0 International License, which permits use, sharing, adaptation, distribution and reproduction in any medium or format, as long as you give appropriate credit to the original author(s) and the source, provide a link to the Creative Commons license, and indicate if changes were made. The images or other third party material in this article are included in the article's Creative Commons license, unless indicated otherwise in a credit line to the material. If material is not included in the article's Creative Commons license and your intended use is not permitted by statutory regulation or exceeds the permitted use, you will need to obtain permission directly from the copyright holder. To view a copy of this license, visit http://creativecommons.org/ licenses/by/4.0/.

(C) The Author(s) 2019 